\title{
COBORDISM INVARIANTS, THE KERVAIRE INVARIANT AND FIXED POINT FREE INVOLUTIONS(1)
}

\author{
BY
}

\section{WILLIAM BROWDER}

\begin{abstract}
Conditions are found which allow one to define an absolute version of the Kervaire invariant in $Z_{2}$ of a Wu- $(q+1)$ oriented $2 q$-manifold. The condition is given in terms of a new invariant called the spectral cobordism invariant. Calculations are then made for the Kervaire invariant of the $n$-fold disjoint union of a manifold $M$ with itself, which are then applied with $M=P^{2 q}$, the real projective space. These give examples where the Kervaire invariant is not defined, and other examples where it has value $1 \in \mathrm{Z}_{2}$. These results are then applied to construct examples of smooth fixed point free involutions of homotopy spheres of dimension $4 k+1$ with nonzero desuspension obstruction, of which some Brieskorn spheres are examples (results obtained also by Berstein and Giffen). The spectral cobordism invariant is also applied directly to these examples to give another proof of a result of Atiyah-Bott. The question of which values can be realized as the sequence of Kervaire invariants of characteristic submanifolds of a smooth homotopy real projective space is discussed with some examples. Finally a condition is given which yields smooth embeddings of homotopy $P^{m}$, s in $R^{m+k}$ (which has been applied by E. Rees).
\end{abstract}

In [7], it was shown how to define an invariant for certain manifolds of even dimension which generalized the Kervaire invariant for framed manifolds. In [8] it was shown how to use this technique to define surgery obstructions. In this paper we shall continue the study of this invariant.

First we consider the question of when the invariant is defined and give much weaker conditions than those employed in [7]. For manifolds of dimension $2 q$ these conditions are shown to be $B\left\langle v_{q+1}\right\rangle$ cobordism invariants (using the notation of [7]). We define a new cobordism invariant called the "spectral cobordism invariant" and show how to determine whether the Kervaire invariant is defined as a function of this invariant. We then study some examples (such as sums of projective spaces), show when the Kervaire invariant is defined and when not, and

Received by the editors March 16, 1972.

AMS (MOS) subject classifications (1970). Primary 57D55, 57D90, 57D65; Secondary 57D40, 57D15, 57E25.

Key words and phrases. Spectral cobordism invariant, $Z_{2}$-valued quadratic forms and their radicals, Arf invariant, surgery obstructions, characteristic submanifolds of smooth homotopy projective spaces, Brieskorn varieties, embeddings.

(1) Part of this paper was written while the author was visiting at the Faculte des Sciences, Orsay, and at the IHES, Bures-sur-Yvette. The author was partially supported by an NSF grant. This paper was originally submitted to Scripta Mathematica on September 24, 1970, and transferred to the Transactions after Scripta temporarily ceased operations. 
use the results to show that (again in the notation of [7]) $\pi_{2 q}\left\langle v_{q+1}\right\rangle=Z_{8}$ generated by $P^{2 q}$. The same examples are used to construct smooth surgery problems, i.e. maps into real projective space $P^{2 q}$ for each $q$, such that the surgery invariants are nonzero, for each $q$. We then construct examples of smooth fixed point free involutions $T$ on $\Sigma^{4 k+1}$ (a generator of $b P^{4 k+2}$ ) such that there are invariant characteristic $\Sigma^{l} \subset \Sigma^{4 k+1}$ if and only if $4 k+1-l \equiv 0$ (4) (an invariant sphere $\Sigma^{l}$ is called characteristic if it is the total inverse image of the standard $S^{l} C$ $S^{4 k+1}$ under an equivariant homotopy equivalence $\left(\Sigma^{4 k+1}, T\right)$ with $\left(S^{4 k+1}, A\right)$, where $A$ is the antipodal map). In particular this gives smooth examples in each dimension $4 k+1$ where the Arf invariant of an involution (see [10]) is nonzero. (This result has been obtained independently by I. Berstein [5] by a direct geometric construction, and by Giffen [13] using the Brieskorn spheres (see \$6).) Such examples were known previously in the piecewise linear case (see [18] and [30]) and in some special dimensions (see [21]). We also study these examples using the spectral cobordism invariant and obtain a lower bound on the number of different examples one can construct this way. Finally we show that these examples are related to the involutions on certain Brieskorn spheres studied by Atiyah and Bott [4] and get a new proof of their result.

In the final section we prove a theorem about smooth embedding of homotopy projective spaces in euclidean space, which has been used by E. Rees [24].

Most of this paper was written before Brown announced his definition of a Kervaire invariant in $Z_{8}$ [11]. His invariant is always defined, unlike ours, but his is not as natural, involving a choice. All the explicit calculations done here could be done with his definition, perhaps more simply.

1. Universal manifolds, cobordism constructions and quadratic forms. In this section we introduce some general constructions for studying a cobordism theory and use them to define a certain $Z_{2}$-valued quadratic form on certain submodules of $H^{q}\left(M ; Z_{2}\right)$ for $M$ a $2 q$-dimensional Poincaré duality space. This is a review of results of [7] in many respects, but, in fact the constructions in [7] are valid for Poincaré duality spaces, while here we shall give a more geometrical definition valid for smooth or p.l. manifolds. In particular we give a somewhat different way to define cobordism invariants in terms of a homotopy construction different from the classical one of Thom. We shall use it later also to study fixed point free involutions. For simplicity we shall always talk about smooth manifolds and linear bundles with the understanding that one can translate the results into the piecewise linear category using micro-bundles [19] or block-bundles ([25], [22]) and p.1. immersion theory [15].

Let us consider closed manifolds $M$ of a given dimension $m$. Let $B$ be a classifying space for a theory of $n$-plane bundles, $n \gg m, \gamma$ the universal 
$n$-plane bundle over $B$. A $B$-orientation of $M$ will mean a linear bundle map of the stable normal bundle $\nu$ of $M^{m} \subset S^{m}+^{n}$ into $\gamma$. If $\nu$ and $\gamma$ were assumed to have additional structure besides linearity, and the bundle map were required to preserve it, then we would have a different notion of $B$-orientation (for example if $\gamma$ and $\nu$ were oriented in the usual sense) (see $[7, \$ 4]$ ). A $B$-cobordism of two $B$ oriented manifolds $M_{0}$ and $M_{1}$ (with orientations $b_{0}: \nu_{0} \rightarrow \gamma, b_{1}: \nu_{1} \rightarrow \gamma$ ) will be a cobordism $W, \partial W=M_{0} \rightarrow M_{1}$, and a bundle map $\bar{b}: \bar{\nu} \rightarrow \gamma$, where $\bar{\nu}$ is the normal bundle of $W \subset S^{m+n} \times I$, with $M_{i} \subset S^{m+n} \times i$, and $\bar{b} \mid \nu_{i}=b_{i}, i=0,1$.

Now let $B_{1}$ be a finite complex with the same homotopy type as $B$ up to dimension $m+2$, for example take $B_{1}=(m+3)$-skeleton of $B$, if $B$ if a complex. Embed $B_{1}$ rectilinearly in $S^{Q}$ for some large $Q$, and take $B_{2}$ to be a regular neighborhood of $B_{1}$ in $S^{Q}$. Let $\gamma_{2}^{-1}$ be the inverse of the pull-back $\gamma_{2}$ of the canonical bundle $\gamma$ to $B_{2}$ and let $W^{s}$ be the total space of the disk bundle of $\gamma_{2}^{-1}$ over $B_{2}$. Then $W$ is a manifold with boundary of dimension $s$. We shall call $W$ a "universal B-manifold." Such a construction was first defined by Milnor [19].

Now $B_{2}$ is parallelizable as it is an open subset of $S^{Q}$, so the tangent bundle of $W$ is $p^{*}\left(\gamma_{2}^{-1}+\epsilon^{Q}\right)$, where $p: W \rightarrow B$ is the projection. It is easy to see that the normal bundle of $W^{s}$ in $S^{s+n}$ is the pull-back of the canonical bundle $\gamma$, to $W$, i.e. $p^{*}\left(\gamma_{2}\right)$.

If $M^{m}$ is a $B$-oriented manifold, $b: \nu \rightarrow \gamma$, then since $W$ is the same homotopy type as $B$ up to dimension $m+2$, we get a unique map $b_{2}: \nu \rightarrow p^{*}\left(\gamma_{2}\right)$ where $\gamma_{2}$ is the pull-back of $\gamma$ to $B_{2}$. Then $b_{2}: \nu \rightarrow p^{*}\left(\gamma_{2}\right)$ defines a unique map $b_{2}^{-1}$ : $\nu^{-1} \rightarrow p^{*}\left(\gamma_{2}\right)^{-1}$, and $\nu^{-1}=\tau+\epsilon^{l}$ the stable tangent bundle of $M$. Hence we get a bundle map $c: \tau+\epsilon^{l}+\epsilon^{Q} \rightarrow p^{*}\left(\gamma_{2}\right)^{-1}+\epsilon^{Q}, c=b_{2}^{-1}+1$, i.e., a linear bundle map of the tangent bundle of $M^{m} \times D^{k}$ in the tangent bundle of $W^{s}, s=m+k$, $k=l+Q$. By Hirsch's Theorem [16] such a bundle map comes from the differential of an immersion $a: M \times D^{k} \rightarrow W$, which is unique up to regular isotopy. As $k$ is very large compared to $m$, we can change $\alpha$ by a regular isotopy to be an embedding on $M$, and hence a small neighborhood of $M$, i.e., on a smaller $M \times D^{k}$. By a similar argument this embedding will be unique up to an isotopy. Thus we have shown

(1.1) Theorem. Let $W^{m+k}$ be the universal manifold constructed above, and $M^{m}$ a closed B-manifold. Then the B-orientation of $M$ defines an embedding $M \times D^{k} C W^{m+k}$ which is unique up to isotopy. Further, a B-cobordism $U$ between $M_{0}$ and $M_{1}$ leads to an embedding of $U^{m+1} \times D^{k} C W^{m+k} \times I$, with restrictions to $M_{i} \times D^{k}$ the given embeddings, for $i=0,1$.

Given a $B$-manifold $M$, and the embedding $M^{m} \times D^{k} \subset W^{m+k}$, we can take the Pontrjagin-Thom construction, i.e., consider the natural collapsing map $g: W / \partial W$ $\rightarrow M \times D^{k} / M \times S^{k-1}=\Sigma^{k} M_{+}$, the $k$-fold reduced suspension of $(M \cup$ point $)$. We 
call $g$ the "spectral orientation map" of $M$.

(1.2) Corollary. If $M^{m}$ is a B-manifold, $W$ a universal manifold as above, then the spectral orientation map $g: W / \partial W \rightarrow \Sigma^{k} M_{+}$is uniquely defined up to bomotopy.

For an isotopy of the embedding leads to a homotopy of $g$.

From the fact that $\Sigma^{k} M_{+}$is $(k-1)$-connected and $W / \partial W$ is of dimension $m+k$, it follows that, since $k$ is larger than $m+1$, all the homotopy information carried by $g$ will be the same, independent of the choice of $B_{1}$ in our construction of $W$.

The spectral orientation map $g$ will play an important role in defining the quadratic form which yields the generalized Kervaire invariant. We recall that in [7] a more general construction was given for $M$ a Poincaré complex, etc., using Spanier-Whitehead duality instead of immersion theory and the Pontrjagin-Thom construction. In the sequel, therefore, we could use this more general situation to enunciate the theorems, thinking of the construction given here as an intuitive special case.

Taking the projection map $M \times D^{k} \rightarrow D^{k}$ and the induced map $t: \Sigma^{k} M_{+} \rightarrow$ $S^{k}$, and composing with $g$,

$$
W / \partial W \stackrel{g}{\rightarrow} \Sigma^{k} M_{+} \stackrel{t}{\rightarrow} S^{k}
$$

we get an element in the cohomotopy group $\pi^{k}(W / \partial W)$. Using the usual transverse regularity argument we then get

(1.3) Corollary. The B-cobordism group of m-manifolds is isomorphic to $\pi^{k}(W / \partial W)$, where $W$ is the universal B-manifold constructed above, dimension $W$ $=m+k, k>m+1$.

This corollary is just the Spanier-Whitehead dual of Thom's theorem that the cobordism group is isomorphic to $\pi_{m+q}\left(T\left(\gamma_{q}\right)\right)$, where $\gamma_{q}$ is the canonical $q$ plane bundle in the $B$-theory.

Instead of taking simply the projection map $M \times D^{k} \rightarrow D^{k}$, let us instead take $c \times 1: M \times D^{k} \rightarrow W \times D^{k}$ where $c: M \rightarrow W$ is the classifying map for the normal bundle of $M$, considering $W$ as the classifying space in dimensions $\leq m$ + 2. The induced map $\Sigma^{k}(c): \Sigma^{k} M_{+} \rightarrow \Sigma^{k} W_{+}$may be composed with $g: W / \partial W \rightarrow$ $\Sigma^{k} M_{+}$to get $b=\left(\Sigma^{k}(c)\right) g: W / \partial W \rightarrow \Sigma^{k} W_{+}$.

(1.4) Corollary. The bomotopy class of $b$ is a cobordism invariant, i.e., if $M_{0}$ is B-cobordant to $M_{1}$ then $b_{0}$ is bomotopic to $b_{1}$.

(We shall call the homotopy class of $b \in\left[W / \partial W, \Sigma^{k} W+\right.$, the spectral cobordism invariant of the manifold M.) 
Proof. From (1.1), we get a map $G: W / \partial W \times I \rightarrow \Sigma^{k} U_{+}$with $G \mid W / \partial W \times i=g_{i}$ the spectral orientation map of $M_{i}, g_{i}: W / \partial W \rightarrow \Sigma^{k} M_{i+}, i=0,1$. Then the classifying maps $c_{i}: M_{i} \rightarrow W$ extend to $C: U \rightarrow W$ with $C / M_{i}=c_{i}, i=0,1$. Thus the composite $\Sigma^{k}(C) \circ G: W / \partial W \times I \rightarrow \Sigma^{k} W_{+}$and clearly induces a homotopy of $g_{0}$ with $g_{1}$.

As $\Sigma^{k} W+$ has $S^{k}$ as a retract, $\left[W / \partial W, \Sigma^{k} W_{+}\right]$has $\pi^{k}(W / \partial W)$ as a retract, and in view of (1.3), $\left[W / \partial W, \Sigma^{k} W\right]$ is in general much larger than the cobordism group. However, the richer structure of $W$ in cohomology, as compared to $S^{k}$, makes it convenient for defining certain cobordism invariants which we shall do in $\$ 2$.

Now let $(U, \partial U)$ be a Poincaré duality pair mod 2 of dimension $n$, i.e., there is a class $[U] \in H_{n}\left(U, \partial U ; Z_{2}\right)$ such that $[U] \cap H^{i}\left(U ; Z_{2}\right) \rightarrow H_{n-i}\left(U, \partial U ; Z_{2}\right)$ is an isomorphism for all $i$. This is easily seen to be equivalent to the statement that the pairing $H^{i}\left(U ; Z_{2}\right) \otimes$ $H^{n-i}\left(U, U ; Z_{2}\right) \rightarrow Z_{2}$, given by $(x, y)=(x \cup y)[U]$, is nonsingular for all $i$. Then the Wu class $v_{i}(U) \in H^{i}\left(U ; Z_{2}\right)$ is defined by the formula $\left(v_{i}(U), x\right)=\left(\mathrm{Sq}^{i} x\right)[U]$, for all $x \in H^{n-i}\left(U, \partial U ; Z_{2}\right)$. It was proved by $W_{u}$ that $v_{i}(U)$ is a certain polynomial in the Steifel-Whitney classes in case $M$ is a manifold, so that a universal Wu class $v_{i} \in H^{i}\left(B_{0} ; Z_{2}\right)$ (or more generally $v_{i} \in H^{i}\left(B_{G} ; Z_{2}\right)$ where $B_{G}$ is classifying space for spherical fibrations). We shall take $B$ to be the classifying space for a bundle theory in which $v_{q+1}=0$. (A universal construction for such a theory is given in $[7, \S 2]$.) Then the construction above, starting from such a $B$, gives a universal manifold $W$ such that $\left(\mathrm{Sq}^{q+1}(x)\right)[W]=0$ for $x \in H^{s-q-1}\left(W, \partial W ; Z_{2}\right)$. (See [7] for a more complete discussion.)

Suppose $M^{2 q}$ is a manifold oriented in a theory where $v_{*+1}=0$ and let $g: W / \partial W \rightarrow \Sigma^{k} M_{+}$be its spectral orientation map. Define $\eta^{*^{q+1}}$ to be the composition

$$
H^{q}\left(M ; Z_{2}\right) \stackrel{\Sigma^{k}}{\longrightarrow} H^{q+k}\left(\Sigma^{k} M_{+} ; Z_{2}\right) \stackrel{g^{*}}{\longrightarrow} H^{q+k}\left(W / \partial W ; Z_{2}\right) .
$$

Following [7] we define a map $\psi:\left(\operatorname{ker} \eta^{*}\right)^{q} \rightarrow Z_{2}$ as follows:

Let $a: M_{+} \rightarrow K\left(Z_{2}, q\right)$ be such that $\alpha^{*}(\iota)=x, \iota \in H^{q}\left(K\left(Z_{2}, q\right) ; Z_{2}\right)$ the fundamental class, $K\left(Z_{2}, q\right)$ the Eilenberg-Mac Lane space with homotopy groups $\pi_{i}=0$ if $i \neq q, \pi_{q}=Z_{2}$. Define $\beta$ to be the composition

$$
W / \partial W \stackrel{g}{\longrightarrow} \Sigma^{k} M_{+} \stackrel{\Sigma^{k} a}{\longrightarrow} \Sigma^{k} K\left(Z_{2}, q\right)
$$

If $\eta^{*}(x)=0$, then $\beta^{*}\left(\Sigma^{k}(\iota)\right)=g^{*}\left(\Sigma^{k} \alpha(\iota)\right)=g^{*} \Sigma^{k}(x)=\eta^{*} x=0$. Hence we may define the functional Steenrod square

$$
\mathrm{Sq}_{\beta}^{q+1}\left(\Sigma^{k}(\iota)\right) \in \frac{H^{2 q+k}\left(W / \partial W ; Z_{2}\right)}{\left(\mathrm{im} \mathrm{Sq} q^{q+1}+\mathrm{im} \beta^{*}\right)} .
$$


It is shown in $\left[7, \S_{1}\right]$ that the denominator $\left(\mathrm{im} \beta^{*}\right)^{2 q+k}=0$, im Sq$q^{q+1}=0$ by assumption, so that $\mathrm{Sq}_{\beta}^{q+1}\left(\Sigma^{k}(\iota)\right) \in H^{2 q+k}\left(W / \partial W ; Z_{2}\right)$. Define $\psi(x)=$ $\left(\mathrm{Sq}_{\beta}^{q+1}\left(\Sigma^{k}(\iota)\right)\right)[W]$. Note that on the right-hand side, only the map $\beta$ depends on $x$. In $\left[7, \S_{1}\right]$, it was shown that if $x, y \in\left(\operatorname{ker} \eta^{*}\right)^{q}$ then $\psi(x+y)=\psi(x)+\psi(y)+$ $(x, y)$, i.e., that $\psi$ is a quadratic form over $Z_{2}$.

2. Definability and additivity of the generalized Kervaire invariant. Let $M^{2 q}$ be a $2 q$-dimensional $(\bmod 2)$ Poincaré duality space and

$$
W / \partial W \stackrel{g}{\longrightarrow} \Sigma^{k} M_{+} \stackrel{\Sigma^{k} c}{\longrightarrow} \Sigma^{k} W_{+}
$$

be maps, with $g$ the spectral orientation map defined by the W-orientation of $M$ and $c$ the classifying map for the Spivak normal fibre space of $M$ (or normal bundle if $M$ is a manifold), $W$ being a "universal manifold" for the fibre space (or bundle) theory with $v_{q+1}=0$ (see $\$ 1$, and [7]). Recall that the homotopy class of $b=$ $\left(\Sigma^{k} c\right) g$ depends only on the cobordism class of $M$, and is called the spectral cobordism invariant of $M$ (see $\$ 1$ ).

Then as in $\oint_{1}$ (see also [7]) we have a quadratic form defined by $\psi$ : $\left(\operatorname{ker} \eta^{*}\right)^{q}$ $\rightarrow Z_{2}$ (where as in $\$ 1, \eta^{*}=g^{*} \Sigma^{k}: H^{q}\left(M ; Z_{2}\right) \rightarrow H^{q+k}\left(W / \partial W ; Z_{2}\right)$ ), and the associated bilinear form

$$
(x, y)=\psi(x+y)-\psi(x)-\psi(y)
$$

is the intersection form $(x, y)=(x \cup y)([M])$, where $[M] \in H_{2 q}\left(M ; Z_{2}\right)$ is the fundamental class. In order to define the Arf invariant of a quadratic form it is necessary that the associated bilinear form be symplectic, that is, that it be nonsingular and $(x, x)=0$ for all $x$. For the bilinear form associated with a quadratic form over $Z_{2}$, the second condition is automatically satisfied, i.e., $(x, x)=$ $\psi(2 x)-2 \psi(x)=0$. In particular it follows that $(x, y)=(y, x)$. But the condition of nonsingularity is not automatic and will not be satisfied in some examples we shall develop later.

If $($,$) is a symmetric bilinear form on a vector space V$, we define the radical $R$ of $($,$) by R=\{r \in V$ such that $(r, x)=0$ all $x \in V\}$. If $\psi$ is a quadratic form $\psi: V \rightarrow Z_{2}$, with $($,$) its associated bilinear form, V$ a $Z_{2}$ vector space, we shall call $\psi$ proper if $\psi(r)=0$ for all $r \in R=$ the radical of $($,$) . If \psi$ is proper, then $\psi$ defines a quadratic form $\psi^{\prime}: V / R \rightarrow Z_{2}$ by $\psi^{\prime}(\bar{x})=\psi(x)$ for $x \in V$ representing $\bar{x} \in V / R$. Then $x+r, r \in R$ also represents $\bar{x} \in V / R$, and $\psi(x+r)=\psi(x)+\psi(r)+(x, r)$. Then $(x, r)=0$ since $r \in R, \psi(r)=0$ since $\psi$ is proper, and thus $\psi^{\prime}: V / R \rightarrow Z_{2}$ is well defined. But the induced bilinear form on $V / R$ is nonsingular so that the Arf invariant of $\psi^{\prime}$ is defined. In this case one may define the Arf invariant of $\psi$ as the Arf invariant of $\psi^{\prime}$, and we have proved (compare [7, (III.1.14)]): 
(2.1) Lemma. The Arf invariant of $\psi$ may be defined if $\psi$ is proper, i.e., if $\psi$ annibilates the radical of its associated bilinear form.

Now we have the following theorem about the geometric situation.

(2.2) Theorem. $c^{*} H^{*}(W)$ is the annibilator of $\left(\operatorname{ker} \eta^{*}\right)$. In particular, if $R$ is the radical of the intersection form on $\left(\operatorname{ker} \eta^{*}\right)^{q}, R=\left(\operatorname{ker} \eta^{*}\right)^{q} \cap c^{*} H^{q}\left(W ; Z_{2}\right)$.

First we prove the following lemma.

(2.3) Lemma. Let $U$ be a compact $n$-manifold with boundary, $i: U \rightarrow W$ be an embedding in the interior of $W$, where $W$ is a compact n-manifold with boundary. Let $g: W / \partial W \rightarrow U / \partial U$ be the natural collapsing map. If $z \in H^{l}(U, \partial U), x \in$ $H^{n-l}(W)$, then

$$
\left(z \cup i^{*} x\right)[U]=\left(g^{*} z \cup x\right)[W]
$$

where $H^{*}(W / \partial W)$ and $H^{*}(U / \partial U)$ are identified with $H^{*}(W, \partial W)$ and $H^{*}(U, \partial U)$ respectively.

Proof of (2.3). Consider the following commutative diagram:

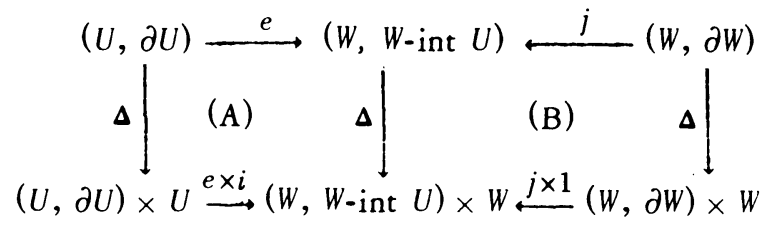

where $e$ is the map of pairs induced by $i, j$ is the natural inclusion, and $\Delta$ is the natural diagonal map in each case, which induce the cup products in cohomology. Now $e$ is an excision, so $e_{*}$ and $e^{*}$ are isomorphisms, and the diagram

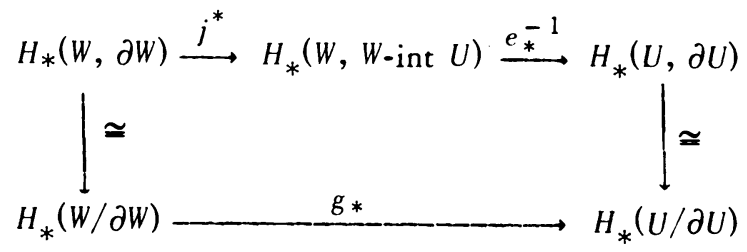

is commutative, together with the similar diagram in cohomology.

Now considering square $(A)$ in the diagram (2.4), we find that $e^{*}\left(e^{*-1}(z) \cup x\right)$ $=e^{*}\left(e^{*-1}(z)\right) \cup i^{*} x=z \cup i^{*} x$. Considering square (B), we get

$$
j^{*}\left(e^{*-1}(z) \cup x\right)=j^{*}\left(e^{*-1}(z)\right) \cup x .
$$

Hence $j^{*} e^{*-1}\left(z \cup i^{*} x\right)=g^{*}\left(z \cup i^{*} x\right)=\left(g^{*} z\right) \cup x$. 
Now $[U]=g_{*}[W]$ so that

$$
\left(z \cup i^{*} x\right)[U]=\left(z \cup i^{*} x\right)\left(g_{*}[W]\right)=\left(g^{*}\left(z \cup i^{*} x\right)\right)[W]=\left(g^{*} z \cup x\right)[W],
$$

which proves (2.3).

Applying (2.3) to the situation of Theorem (2.2), we let $U=M \times D^{k}, W=W$ so that $i=c$, and $g=g$. Then if $x \in H^{*}(W)$ and $y \in H^{*}(M)$, we have

$$
\begin{aligned}
\left(y, c^{*} x\right) & =\left(y \cup c^{*} x\right)[M]=\left(\Sigma^{k} y \cup c^{*} x\right)\left[M \times D^{k}\right] \\
& =\left(g^{*} \Sigma^{k} y \cup x\right)[W]=\left(\eta^{*} y \cup x\right)[W] .
\end{aligned}
$$

Hence it follows that $\left(\operatorname{ker} \eta^{*}\right)$ annihilates $\left(\operatorname{im} c^{*}\right)$, so $\left(\operatorname{im} c^{*}\right) \subset \mathbb{Q}=$ annihilator of $\left(\operatorname{ker} \eta^{*}\right)$.

Since $H^{*}(M) \cong \operatorname{ker} \eta^{*}+\operatorname{im} \eta^{*}$, it follows that rank $\left(\operatorname{im} c^{*}\right) \leq \operatorname{rank}\left(\operatorname{im} \eta^{*}\right)=$ rank $\mathcal{A}$. Similarly, $\left(\operatorname{im} \eta^{*}\right) \subset$ annihilator of $\left(\operatorname{ker} c^{*}\right)$ so rank (im $\left.\eta^{*}\right) \leq$ rank $\left(\operatorname{im} c^{*}\right)=$ rank of the annihilator of ker $c^{*}$. Hence rank $\left(\operatorname{im} \eta^{*}\right)=$ rank (im $\left.c^{*}\right)$ and it follows that $c^{*} H^{*}(W)=\mathbb{Q}$, the annihilator of (ker $\left.\eta^{*}\right)$. Then $R=\mathcal{G} \cap\left(\operatorname{ker} \eta^{*}\right)^{q}=c^{*} H^{q}(W) \cap\left(\operatorname{ker} \eta^{*}\right)^{q}$, and (2.2) is proved.

(2.6) Corollary. The Arf invariant of $\psi$ is well defined on $\left(\operatorname{ker} \eta^{*}\right)^{q}$ if and only if $\psi \mid c^{*} H^{q}\left(W ; Z_{2}\right) \cap\left(\operatorname{ker} \eta^{*}\right)^{q}=0$.

We recall that $\psi$ is defined in $\$ 1$ using the functional $\mathrm{Sq}^{q+1}$ associated to the composite

$$
W / \partial W \stackrel{g}{\longrightarrow} \Sigma^{k} M_{+} \stackrel{\Sigma^{k} a}{\longrightarrow} \Sigma^{k} K\left(Z_{2}, q\right)
$$

where $x=a^{*}(\iota) \in\left(\operatorname{ker} \eta^{*}\right)^{q}$. If $x=c^{*}(z), z \in H^{q}\left(W ; Z_{2}\right)$, then $a$ factors through $\delta: W \rightarrow K\left(Z_{2}, q\right)$, so that $\alpha=\delta c$, and we have

$$
W / \partial W \stackrel{g}{\longrightarrow} \Sigma^{k} M_{+} \stackrel{\Sigma^{k} c}{\longrightarrow} \Sigma^{k} W_{+} \stackrel{\Sigma^{k} \delta}{\longrightarrow} \Sigma^{k} K\left(Z_{2} q\right)
$$

Now $\left(\Sigma^{k} c\right) g=b$, the map defined in $\$ 1$, whose homotopy class is the spectral cobordism invariant of $M$.

Thus, if $x \in\left(\operatorname{ker} \eta^{*}\right)^{q} \cap c^{*} H^{q}\left(W ; Z_{2}\right), x=c^{*} y$, the map $\beta: W / \partial W \rightarrow$ $\Sigma^{k} K\left(Z_{2}, q\right)$ corresponding to $x$ is the composition $\beta=\left(\Sigma^{k} \delta\right) b$, where $b: W / \partial W \rightarrow$ $\Sigma^{k} W_{+}$is the spectral cobordism invariant of $M, \delta^{*}(\iota)=y$, and $b^{*} y=0$. Thus $\mathrm{Sq}_{\beta}^{q+1}\left(\Sigma^{k}(\iota)\right)=\mathrm{Sq}_{\left(\Sigma^{k} \delta\right) b}^{q+1}\left(\Sigma^{k}(\iota)\right)$ and it follows that $\psi(x)=0$ for all $x \epsilon$ $\left(\operatorname{ker} \eta^{*}\right)^{q} \cap c^{*} H^{q}\left(W ; Z_{2}\right)$ if and only if $\left(\mathrm{Sq}_{\left(\Sigma^{k} \delta\right) b}^{q+1}\left(\Sigma^{k}(\iota)\right)\right)[W]=0$ for all $\delta: W_{+} \rightarrow$ $K\left(Z_{2}, q\right)$, such that $\delta^{*} \iota \in \operatorname{ker} b^{*} \subset H^{q+k}\left(\Sigma^{k} W ; Z_{2}\right)$. This condition depends only on $b$, so we get the following theorem. 
(2.7) Theorem. Let $M^{2 q}$ be a Poincaré duality complex of dimension $2 q$, oriented in a theory which bas $v_{q+1}=0$. Then for the quadratic form $\psi:\left(\operatorname{ker} \eta^{*}\right)^{q}$ $\rightarrow Z_{2}$, defined by the orientation, the Arf invariant of $\psi$ is defined if and only if $\left(\mathrm{Sq}_{\left(\Sigma^{k} \delta\right) b}^{q+1}\left(\Sigma^{k} \iota\right)\right)[W]=0$ for all $\delta: W_{+} \rightarrow K\left(Z_{2}, q\right)$ sucb that $\delta^{*} \iota \in \operatorname{ker} b^{*} \Sigma^{b} \cap$ $H^{q}\left(W_{+} ; Z_{2}\right)$, where $b: W / \partial W \rightarrow \Sigma^{k} W_{+}$is the spectral cobordism invariant of $M$ (see $\$ 1)$. In particular, the property that the Arf invariant is defined is a cobordism invariant (in a theory with $v_{q+1}=0$ ).

If the hypothesis of (2.7) is satisfied then we set $k(M)=$ Arf invariant of $\psi$, and we call it the Kervaire invariant of M. By [7, (3.2)], this is exactly Kervaire's invariant in the case of framed manifolds.

(2.8) Corollary. With $M^{2 q}$ as in (2.7), suppose also that $b^{*}\left(H^{2 q+k}\left(\Sigma^{k} W_{+} ; Z_{2}\right)\right)$ $=0$. Then $k(M)$ is defined if and only if $\mathrm{Sq}_{b}^{q+1}\left(\Sigma^{k} y\right)[W]=0$ for every $y \epsilon$ $\operatorname{ker} b^{*} \Sigma^{k} \cap H^{q}\left(W_{+} ; Z_{2}\right)$.

Proof. Note that if $\delta^{*} \iota=y$, then

$$
\mathrm{Sq}_{\left(\Sigma^{k} \delta\right) b}^{q+1}\left(\Sigma^{k} \iota\right)=\mathrm{Sq}_{b}^{q+1}\left(\Sigma^{k} y\right) \quad \bmod b^{*}\left(H^{2 q+k}\left(\Sigma^{k} W_{+} ; Z_{2}\right)\right)=0
$$

Hence the equality holds and (2.8) follows from (2.7).

Now let us consider the operation of addition in our cobordism theory, which is represented by taking the disjoint union of the manifolds, so that $M_{1} \cup M_{2}$ represents the sum of $M_{1}$ and $M_{2}$. Suppose we have $i_{1}: M_{1} \times D^{k} \subset W$ and $i_{2}: M_{2} \times$ $D^{k} \subset W$. Then we may consider $i_{1} \times 1 / 3: M_{1} \times D^{k}+1 \subset W \times I$ and $i_{2} \times 2 / 3: M_{2} \times$ $D^{k+1} \subset W \times I$, so that the embeddings are disjoint in $W \times I$ and $W \times I$ is still a universal manifold. Then the spectral orientation $g: W \times I / \partial(W \times I) \rightarrow$ $\Sigma^{k+1}\left(\left(M_{1} \cup M_{2}\right)_{+}\right)$can be factored through $W \times I / \partial(W \times I) \rightarrow W \times I /(\partial(W \times I) \cup W \times 1 / 2)$, and $W \times I /(\partial(W \times I) \cup W \times 1 / 2)=(W \times I / \partial(W \times I)) \vee(W \times I / \partial(W \times I))$. In fact $W \times$ $I / \partial(W \times I)=\Sigma(W / \partial W)$ and this is the natural map $\Sigma(W / \partial W) \rightarrow \Sigma(W / \partial W) \vee \Sigma(W / \partial W)$. Also $\left(M_{1} \cup M_{2}\right)_{+}=M_{1+} \vee M_{2+}$, so $\Sigma^{k+1}\left(M_{1} \cup M_{2}\right)_{+}=\Sigma^{k+1} M_{1+} \vee \Sigma^{k+1} M_{2+}$ and $g: \Sigma(W / \partial W) \rightarrow \Sigma^{k+1} M_{1+} \vee \Sigma^{k+1} M_{2+}$ is $g=g_{1}^{\prime}+g_{2}^{\prime}$, where $g_{i}^{\prime}$ is the composite $\Sigma(W / \partial W) \stackrel{\Sigma g_{i}}{\longrightarrow} \Sigma^{k+1} M_{i+} \rightarrow \Sigma^{k+1} M_{1+} \vee M_{2+}, g_{i}$ being the spectral orientation for $M_{i}$, and + is the addition of maps induced by the suspension structure of $\Sigma(W / \partial W)$. Now we return to the previous notation with $g: W / \partial W \rightarrow \Sigma^{k}\left(M_{1} \cup M_{2}\right)_{+}$, with the understanding that everything is a suspension, $g=g_{1}^{\prime}+g_{2}^{\prime}$ etc. as above, and we have shown:

(2.9) Lemma. Let $g_{i}: W / \partial W \rightarrow \Sigma^{k} M_{i+}$ be the spectral orientation maps for $M_{i}, i=1$, 2. Then the spectral orientation map for $M_{1} \cup M_{2}$ is given by $g=g_{1}^{\prime}+$ $g_{2}^{\prime}\left(g_{j}^{\prime}=i_{j} g_{j}\right.$, where $i_{j}$ is the inclusion $i_{j}: \Sigma^{k} M_{j+} \rightarrow\left(\Sigma^{k} M_{1+}\right) \vee\left(\Sigma^{k} M_{2+}\right), j=1,2$, 
and $\left.\Sigma^{k} c=\Sigma^{k} c_{1} \vee \Sigma^{k} c_{2}\right)$. Hence $b=b_{1}+b_{2}$ and the spectral cobordism invariant is additive on cobordism classes.

Since $\operatorname{ker}\left(b_{1}^{*}+b_{2}^{*}\right)=\operatorname{ker}\left(b_{1}+b_{2}\right)^{*}$ is larger in general than $\operatorname{ker} b_{1}^{*}+\operatorname{ker} b_{2}^{*}$, the condition that the Kervaire invariant is defined for two out of three for $M=$ $M_{1}+M_{2}$ is not enough to ensure it is defined for the third. We will see some examples of this in $\$ 3$. In fact we will give examples of $M_{1}$ and $M_{2}$, where (a) it is defined for $M_{1}$ and $M_{2}$ and not for $M_{1}+M_{2}$, (b) it is not defined for $M_{1}$ or $M_{2}$ and defined for $M_{1}+M_{2}$, (c) it is defined for $M_{1}$, not for $M_{2}$, and either defined or not for $M_{1}+M_{2}$. Thus all possible situations occur.

Hence in order to state an addition theorem for the Kervaire invariant we must make assumptions that it is defined for each space. We give an additivity theorem below (2.13), but it is unclear what are the most general hypotheses under which additivity holds.

Let $M_{1}$ and $M_{2}$ be Poincaré duality spaces $(\bmod 2)$, oriented in a theory where $v_{q+1}=0, M=M_{1}+M_{2}$ (notation as above).

By (2.9), $g=g_{1}^{\prime}+g_{2}^{\prime}: W / \partial W \rightarrow \Sigma^{k} M_{1+} \vee \Sigma^{k} M_{2+}$, where $g_{i}^{\prime}$ is the spectral orientation $g_{i}: W / \partial W \rightarrow \Sigma^{k} M_{i+}$ followed by the inclusion in $\Sigma^{k} M_{i+} \vee \Sigma^{k} M_{2+}$. It follows that $\left(\operatorname{ker} g^{*}\right) \supseteq\left(\operatorname{ker} g_{1}^{*}\right)+\left(\operatorname{ker} g_{2}^{*}\right)$, and in general the inclusion may be strict (which causes all the difficulties, in the definability of the Kervaire invariant being nonadditive, and in proving additivity).

Let $c:\left(M_{1} \cup M_{2}\right)_{+} \rightarrow W_{+}$be the classifying map for $M_{1} \cup M_{2}$ so that $c \mid M_{i}=c_{i}$, the classifying map for $M_{i}, \Sigma^{k} c: \Sigma^{k} M_{1+} \vee \Sigma^{k} M_{2+} \rightarrow \Sigma^{k} W_{+}$is the sum $\Sigma^{k} c^{i}=\Sigma^{k} c_{1}+\Sigma^{k} c_{2}$. Then $\operatorname{im} c^{*}=\operatorname{im}\left(c_{1}^{*}+c_{2}^{*}\right)$, but note that of course this is not the sum of the two images, in general.

Set $\eta^{*}=g^{*} \Sigma^{k}$, as usual, so that $\eta^{*}=\eta_{1}^{*}+\eta_{2}^{*}$, where $\eta_{i}^{*}=g_{i}^{*} \Sigma^{k}$, and as above $\left(\operatorname{ker} \eta^{*}\right) \supseteq\left(\operatorname{ker} \eta_{1}^{*}\right)+\left(\operatorname{ker} \eta_{2}^{*}\right)$.

Let $\psi$ be the quadratic form for $M_{1}+M_{2}, \psi_{i}$ the quadratic form for $M_{i}$, $i=$ 1,2 (as in $\$ 1$ ).

(2.10) I emma. On $\left(\operatorname{ker} \eta_{1}^{*}\right)^{q}+\left(\operatorname{ker} \eta_{2}^{*}\right)^{q}, \psi=\psi_{1}+\psi_{2}$.

Proof. Since $H^{*}\left(M_{1} ; Z_{2}\right)$ and $H^{*}\left(M_{2} ; Z_{2}\right)$ are orthogonal in $H^{*}\left(M ; Z_{2}\right)$, it follows from the fact that $\psi$ is quadratic that $\psi\left(x_{1}+x_{2}\right)=\psi\left(x_{1}\right)+\psi\left(x_{2}\right)$ if $x_{i} \epsilon$ $\operatorname{ker} \eta_{i}^{*}, i=1,2$. If $f: M \rightarrow K\left(Z_{2}, q\right)$ is such that $f^{*}(\iota)=x_{i} \in \operatorname{ker} \eta_{i}^{*}, i=1$ or 2 , then $f$ factors: $M \stackrel{d_{i}}{\rightarrow} M_{i} \stackrel{f_{i}}{\rightarrow} K\left(Z_{2}, q\right)$ where $d_{i}: M \rightarrow M_{i}$ is the identity on $M_{i}$ and the constant map on the other component, $f_{i}^{*}(\iota)^{i}=x_{i} \in H^{q}\left(M_{i} ; Z_{2}\right)$. Then $\left(\Sigma^{k} d_{i}\right) g=$ $\left(\Sigma^{k} d_{i}\right)\left(g_{1}^{\prime}+g_{2}^{\prime}\right)=g_{i}$, the spectral orientation for $M_{i}$ (using (2.9)) and hence $g\left(\Sigma^{k} f\right)=g_{i} f_{i}$ and $\mathrm{Sq}_{g\left(\Sigma^{k} f\right)}^{q+1}\left(\Sigma^{k} \iota\right)=\mathrm{Sq}_{g_{i} f_{i}}^{q+1}\left(\Sigma^{k} \iota\right)$ and hence $\psi\left(x_{i}\right)=\psi_{i}\left(x_{i}\right)$ and the lemma follows.

We get as a simple consequence 
(2.11) Proposition. If $\left(\operatorname{ker} \eta^{*}\right)^{q}=\left(\operatorname{ker} \eta_{1}^{*}\right)^{q}+\left(\operatorname{ker} \eta_{2}^{*}\right)^{q}$, and if the Kervaire invariant is defined for $M_{1}$ and $M_{2}$, then it is defined for $M_{1}+M_{2}$ and $k\left(M_{1}+M_{2}\right)=k\left(M_{1}\right)+k\left(M_{2}\right)$.

Proof. The radical is the sum of the radicals, so $\psi=\psi_{1}+\psi_{2}$ annihilates the radicals, and $k\left(M_{1}+M_{2}\right)$ is defined. Hence, from $(2.10), k\left(M_{1}+M_{2}\right)=k\left(M_{1}\right)+$ $k\left(M_{2}\right)$.

(2.12) Corollary. If $\eta_{i}^{*} H^{q}\left(M_{i} ; Z_{2}\right)=0, i=1,2$, then $k$ is defined for $M_{1}$, $M_{2}$, and $M_{1}+M_{2}$ and is additive, i.e., $k\left(M_{1}+M_{2}\right)=k\left(M_{1}\right)+k\left(M_{2}\right)$.

We would like to have more delicate results than (2.11), but it seems difficult to obtain a general theorem. However, we may prove

(2.13) Theorem. Suppose the Kervaire invariant is defined for $M_{1}$ and $M_{2}$, and that $b_{2}^{*}=0$ on $H^{q}\left(W ; Z_{2}\right)$. Then the Kervaire invariant is defined for $M_{1}+$ $M_{2}$ and $k\left(M_{1}+M_{2}\right)=k\left(M_{1}\right)+k\left(M_{2}\right)$.

(2.14) Corollary. Suppose $k\left(M_{1}\right), k\left(M_{2}\right)$, and $k\left(M_{1}+M_{2}\right)$ all defined and suppose $b_{1}^{*}=b_{2}^{*}$ on $H^{q}\left(W ; Z_{2}\right)$. Then $k\left(M_{1}+M_{2}\right)=k\left(M_{1}\right)+k\left(M_{2}\right)$.

Proof of Corollary (2.14). Under the hypothesis, $b^{*}=b_{1}^{*}+b_{2}^{*}=2 b_{1}^{*}=0$ on $H^{q}\left(W ; Z_{2}\right)$. Hence by $(2.13)$, we have $k\left(-M_{1}+\left(M_{1}+M_{2}\right)\right)=k\left(-M_{1}\right)+k\left(M_{1}+M_{2}\right)$. But $k\left(-M_{1}\right)=k\left(M_{1}\right)$, obviously, and $-M_{1}+\left(M_{1}+M_{2}\right)=M_{2}$. Hence we have $k\left(M_{1}\right)=k\left(M_{1}\right)+k\left(M_{1}+M_{2}\right)$, and shifting $k\left(M_{1}\right)$ to the other side of the equation gives the result.

Note that in general - $M$ may not be cobordant to $M$ in a cobordism theory with $v_{q+1}=0$, as we shall see in $\$ 3$ for $P^{2 q}$, the real projective space. But the spectral orientation and classifying maps differ by automorphisms, so that the definability and value of the Kervaire invariant is the same for $M$ and $-M$.

(2.15) Corollary. Let $M^{2 q}$ be such that (image $\left.c^{*}\right)^{q} C\left(\operatorname{ker} \eta^{*}\right)^{q}$ and $k(M)$ is defined. Then $k(n M)$ is defined for all $n$ and $k(n M)=n k(M)$.

Proof. (image $\left.c^{*}\right)^{q} \subset\left(\operatorname{ker} \eta^{*}\right)^{q}$ implies that $b^{*}=\eta^{*} c^{*}=0$, and hence $b_{n}^{*}=$ $n b^{*}=0$, where $b_{n}$ is the spectral cobordism invariant for $n M$. Since $k(M)$ is defined, Theorem (2.13) applies to prove $(2.15)$ by a simple induction.

Proof of Theorem (2.13). Since $b_{2}^{*}=0$ on $H^{q}\left(W ; Z_{2}\right)$ and $k\left(M_{2}\right)$ is defined, it follows that $\mathrm{Sq}_{r}^{q+1}\left(\Sigma^{k} \iota\right)[W]=0$ for $r=\left(\Sigma^{k} \alpha\right) b_{2}$, any $\alpha: W_{+} \rightarrow K\left(Z_{2}, q\right)$. Since $k\left(M_{1}\right)$ is defined, $\mathrm{Sq}_{t}^{q+1}\left(\Sigma^{k} \iota\right)[W]=0$ for $t=\left(\Sigma^{k} \beta\right) b_{1}, \beta: W_{+} \rightarrow K\left(Z_{2}, q\right)$ such that $\beta^{*}(\iota) \in\left(\operatorname{ker} b_{1}^{*}\right)^{q}$.

Now $b_{2}^{*}=0$, and $b=b_{1}+b_{2}$ implies that $\left(\operatorname{ker} b^{*}\right)^{q}=\left(\operatorname{ker} b_{1}^{*}\right)^{q}$. Now $\left(\Sigma^{k} \beta\right)\left(b_{1}+b_{2}\right)=\left(\Sigma^{k} \beta\right) b_{1}+\left(\Sigma^{k} \beta\right) b_{2}$, so that if $x \in\left(\operatorname{ker} b_{1}^{*}\right)^{q}, \alpha=\beta: W_{+} \rightarrow$ $K\left(Z_{2}, q\right)$ such that $\alpha^{*}(\iota)=\beta^{*}(\iota)=x$, then $\psi(x)=\left(\operatorname{Sq}_{r+t}^{q+1}\left(\Sigma^{k}(\iota)\right)\right)[W]=$ $\left(\mathrm{S}_{r}^{q+1}\left(\Sigma^{k} \iota\right)+\mathrm{Sq}_{t}^{q+1}\left(\Sigma^{k}(\iota)\right)[W]=0\right.$ using $\left.[7,(1.6))\right]$. Hence $k\left(M_{1}+M_{2}\right)$ is defined, by (2.7). 
Now $\operatorname{ker} \eta^{*} \supset \operatorname{ker} \eta_{1}^{*}+\operatorname{ker} \eta_{2}^{*}$, and if we set $R_{i}=\operatorname{ker} \eta_{i}^{*} \cap$ image $c_{i}^{*}=$ radical of $($,$) on \operatorname{ker} \eta_{i}^{*}$, by (2.2), then $\operatorname{ker} \eta_{i}^{*}=R_{i}+B_{i}$, where $($,$) is nonsingular on$ $B_{i}, i=1,2$. Then we may choose a complementary subspace $N$ to $\operatorname{ker} \eta_{1}^{*}+\operatorname{ker} \eta_{2}^{*}$ in ker $\eta^{*}$ such that $N$ is orthogonal to $B_{1}+B_{2}$ (by subtracting off elements of $B_{1}+B_{2}$, if necessary). Then the Arf invariant $c(\psi)=c\left(\psi_{1}\right)+c\left(\psi_{2}\right)+c\left(\psi^{\prime}\right)$ where $\psi^{\prime}$ is $\psi \mid\left(R_{1}+R_{2}+N\right)$. Thus to prove the theorem we must show $c\left(\psi^{\prime}\right)=0$.

Since $b_{2}^{*}=\eta_{2}^{*} c_{2}^{*}=0$, it follows that im $c_{2}^{*} \subset \operatorname{ker} \eta_{2}^{*}$, so that $R_{2}=\operatorname{im} c_{2}^{*}$.

Now $\eta_{2}^{*}$ is injective on $N$. For if $(x, y) \in N \subset H^{q}\left(M_{1} ; Z_{2}\right)+H^{q}\left(M_{2} ; Z_{2}\right)$, and $\eta_{2}^{*}(x, y)=0$, i.e., $y \in \operatorname{ker} \eta_{2}^{*}$, then $\eta^{*}(x, y)=\eta_{1}^{*} x+\eta_{2}^{*} y=\eta_{1}^{*} x=0$ so $x \in \operatorname{ker} \eta_{1}^{*}$ and $(x, y) \in \operatorname{ker} \eta_{1}^{*}+\operatorname{ker} \eta_{2}^{*}$. But $N \cap\left(\operatorname{ker} \eta_{1}^{*}+\operatorname{ker} \eta_{2}^{*}\right)=0$, so $(x, y)=0$ and $\eta_{2}^{*}$ is injective on $N$. Then it follows from (2.5) that $z \rightarrow(z):, N \rightarrow Z_{2}$ defines a map of $R_{2}=\mathrm{im} c_{2}^{*}$ onto $\operatorname{Hom}\left(N, Z_{2}\right)$ so that $N \cap R=0$, where $R=$ radical of $($,$) on \operatorname{ker} \eta^{*}$. Since $\psi^{\prime} \mid R_{1}+R_{2} \equiv 0$ and $R_{1}+R_{2}$ is a self-annihilating subspace under (, ), it follows that we may choose representatives $a_{i}, b_{i} \in \operatorname{ker} \eta^{*}$ of a simplectic basis of $\operatorname{ker} \eta^{*} \mid R$ so that $a_{i} \in \operatorname{im~} c_{2}^{*}=R_{2}, b_{i} \in N$. Since $\psi^{\prime} \mid R_{2}$ $\equiv 0$, it follows that $c\left(\psi^{\prime}\right)=0$, which completes the proof of (2.13).

We note in passing the following property of $h$.

(2.16) Proposition. Let $x \in H^{*}(W ; F), F$ a field. Then $b^{*} x=0$ if and only if all characteristic numbers involving $x$ are zero.

Proof. Suppose $y \in H^{*}(W ; F)$ such that $\left(c^{*} x, c^{*} y\right)=\left(\left(c^{*} x\right) \cup\left(c^{*} y\right)\right)[M] \neq 0$. By (2.5), $\left(c^{*} x, c^{*} y\right)=\left(\eta^{*} c^{*} x, y\right)=\left(\eta^{*} c^{*} x \cup y\right)[W] \neq 0$. Hence $b^{*} x=\eta^{*} c^{*} x \neq 0$.

Suppose $b^{*} x \neq 0$. By Poincaré duality in $W$, there is a $y \in H^{*}(W ; F)$ such that $\left(b^{*} x, y\right) \neq 0$. But $b^{*} x=\eta^{*} c^{*} x$, and $\left(\eta^{*} c^{*} x, y\right)=\left(c^{*} x, c^{*} y\right)$ by (2.5) so $\left(c^{*} x \cup c^{*} y\right)[M] \neq 0$.

Thus the induced cohomology map of the spectral cobordism invariant $b$ may be interpreted in terms of characteristic numbers. But more subtle properties of $b$, such as the functional $\mathrm{Sq}^{q+1}$ of $\left(\Sigma^{k} \alpha\right) b, \alpha_{:} w_{+} \rightarrow K\left(Z_{2}, q\right)$, may be harder to interpret in terms of classical invariants.

3. Multiples of a manifold. In this section we study multiples of a fixed manifold $M$. By (2.14) if $k(M)$ and $k(2 M)$ are defined then $k(2 M)=2 k(M)=0$, and by (2.14), $k(n M)=n k(M)$, so we wish to study the question: When is $k(2 M)$ defined? We will give a condition (which is independent of whether $k(M)$ is defined or not): $k(2 M)$ is defined if and only if, in $M, v_{q}(M) \in \operatorname{ker} \eta^{*}$, or equivalently, if and only if all characteristic numbers of $M$ involving $v_{q}(M)$ are zero (Corollary (3.9)). Next we consider how to calculate $k(2 M)$ when $k(M)$ is not defined and we show that if $k(M)$ is not defined but $k(2 M)$ is defined, then $k(2 M)=\psi\left(v_{q}(M)\right.$ ) (calculated in $M)$, (Theorem (3.10)). This yields the corollary (3.11) that $k(4 M)$ is defined and $k(4 M)=\left(v_{q}(M)\right)^{2}[M]$. (It follows that any $M^{2 q}$ with $\left(v_{q}(M)\right)^{2}[M] \neq 0$ has order 8 in 
the $B_{0}\left\langle v_{q+1}\right\rangle$-cobordism group $\pi_{2 q}\left\langle v_{q+1}\right\rangle$, in the notation of [7].)

Our results in this section will be based on the following: Let $f: X \rightarrow$ $K\left(Z_{2}, q\right)$ such that $f^{*}(\iota)=x$. Then $\Sigma f: \Sigma X \rightarrow \Sigma K\left(Z_{2}, q\right)$ is the suspension of $f$ and $2 \Sigma f$ is the sum of $\Sigma f$ with itself using the suspension structure of $\Sigma X$.

(3.1) Theorem. The functional square $\mathrm{Sq}_{2 \Sigma f}^{q+1}$ is defined and bas indeterminacy 0 , so that $\mathrm{Sq}_{2 \Sigma f}^{q+1}: H^{q+1}\left(\Sigma K\left(Z_{2}, q\right) ; Z_{2}\right) \rightarrow H^{2 q+1}\left(\Sigma X ; Z_{2}\right)$, and $\mathrm{Sq}_{2 \Sigma j}^{q+1}(\Sigma(\iota))=\Sigma\left(x^{2}\right)$.

Proof. Recall that if $a, b: \Sigma X \rightarrow Y,(a+b)^{*}=a^{*}+b^{*}$, so that with $Z_{2}$ coefficients, $(2 a)^{*}=2\left(a^{*}\right)=0$, and image $(2 a)^{*}=0$ in $Z_{2}$-cohomology. Further $\mathrm{Sq}^{q+1}\left(\Sigma_{\iota}\right)=\Sigma \mathrm{Sq}^{q+1}(\iota)=0$ since dimension $\iota=q$ and $\mathrm{Sq}^{q+1}$ annihilates elements of dimension $<q+1$. Hence $\mathrm{Sq}_{2 \Sigma}^{q+1}$ is defined on $\Sigma_{\iota}$.

In $\Sigma X, \mathrm{Sq}^{q+1} \Sigma=\Sigma \mathrm{Sq}^{q+1}$ implies Sq ${ }^{q+1}: H^{q}\left(\Sigma X ; Z_{2}\right) \rightarrow H^{2 q+1}\left(\Sigma X ; Z_{2}\right)$ is zero, for the same dimension reason. Since image $(2 \Sigma f)^{*}=0$, as above, it follows that the indeterminacy of the functional $\mathrm{Sq}^{q+1}$ is zero (compare [7, (1.1)]).

To calculate the value of $\mathrm{Sq}_{2 \Sigma f}^{q+1}(\Sigma(\iota))$ we consider the map $2 f: X \rightarrow K\left(Z_{2}, q\right)$ defined as follows:

$$
X \stackrel{\Delta}{\longrightarrow} X \times X \stackrel{f \times f}{\longrightarrow} K\left(Z_{2}, q\right) \times K\left(Z_{2}, q\right) \stackrel{\mu}{\longrightarrow} K\left(Z_{2}, q\right)
$$

where $\Delta(x)=(x, x), x \in \lambda^{\prime}$ is the diagonal, $\mu^{*}(\iota)=\iota \otimes 1+1 \otimes \iota$, so $\mu$ defines the $H$-space structure of $K=K\left(Z_{2}, q\right)$. Clearly $(2 f)^{*}(\iota)=2 x=0$ since we have $Z_{2}$ coefficients and hence $2 f$ is homotopic to a constant.

Suspending the sequence (3.2) and utilizing the homotopy equivalences $\Sigma(X \times X) \cong \Sigma X \vee \Sigma X \vee \Sigma(X \wedge X)$ and $\Sigma(K \times K) \cong \Sigma K \vee \Sigma K \vee \Sigma(K \wedge K)$ (cf. $[7,(1.5)])$, we get a commutative diagram:

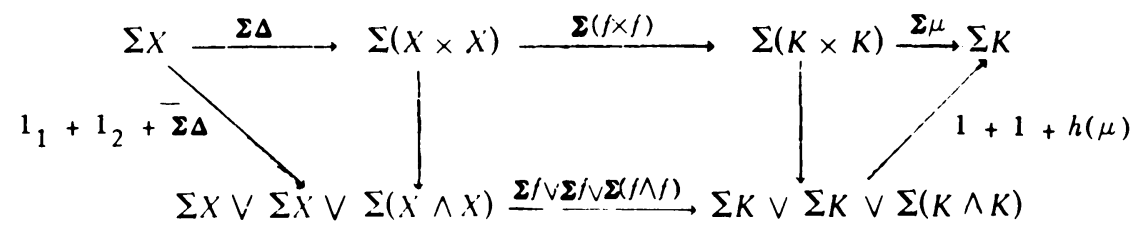

where $1_{i}: \Sigma X \rightarrow \Sigma X \vee \Sigma X^{\prime} \vee \Sigma(X \wedge X)$ is the inclusion into the $i$ th term of the wedge, $i=1,2, \bar{\Delta}=s \Delta, s: X \times X \rightarrow X \wedge X$ is the natural map collapsing $X \vee X$ to a point, and $b(\mu): \Sigma(K \wedge K) \rightarrow \Sigma K$ is the Hopf construction on $\mu$ (cf. [7, (1.5) and after]).

Recall that for a suspension map $\omega=\Sigma a, \Sigma \Lambda \stackrel{\omega}{\rightarrow} \Sigma_{B} \stackrel{\phi, \psi}{\longrightarrow} C$ we have that $(\phi+\psi) \omega=\phi \omega+\psi \omega$ (which is not true for an arbitrary map $\omega)$. Hence the composite map in (3.3) along the lower level gives us $\Sigma(2 f) \cong \Sigma f+\Sigma f+$ $h(\mu)(\Sigma(f \wedge f))(\Sigma \Delta)$. Now $2 f \cong 0$ implies $\Sigma(2 f) \cong 0$ and thus we have shown

$$
2(\Sigma f) \cong-b(\mu)(\Sigma(f \wedge f))(\Sigma \Delta) \text {. }
$$


(3.5) Lemma. Let $A \stackrel{a}{\rightarrow} B \stackrel{b}{\rightarrow} C$ be maps. If $\mathrm{Sq}_{b}^{q+1}(x)$ and $\mathrm{Sq}_{b a}^{q+1}$ are defined with indeterminacy zero, then

$$
\mathrm{Sq}_{b a}^{q+1}(x)=a^{*} \mathrm{Sq}_{b}^{q+1}(x)
$$

The proof is routine from the definitions.

Now we recall that by the argument of $[26,(5.3)]$ we have

$$
\mathrm{Sq}_{h(\mu)}^{q+1}(\Sigma(\iota))=\Sigma(\iota \wedge \iota)
$$

If $\phi: \Sigma X \rightarrow Y,-\phi$ is the map $\phi \circ \tau$ where $\tau: \Sigma X \rightarrow \Sigma X$ is given by $\tau(x, t)=$ $(x,-t)$, for $\Sigma X=X \times I /(X \times 0 \cup X \times 1)$, so $\tau^{*}=(-1)$. Hence by (3.5), $\mathrm{Sq}_{-\phi}^{q+1}=$ $\tau^{*} \mathrm{Sq}_{\phi}^{q+1}=\mathrm{Sq}_{\phi}^{q+1}$. Therefore we have from (3.4), (3.5) and (3.6)

$$
\begin{aligned}
\mathrm{Sq}_{2(\Sigma f)}^{q+1} & (\Sigma \iota)=\mathrm{Sq}_{b(\mu)(\Sigma(f \wedge f))(\Sigma \bar{\Delta})}^{q+1}(\Sigma \iota) \\
& =(\Sigma \bar{\Delta})^{*}(\Sigma(f \wedge f))^{*} \mathrm{Sq}_{b(\mu)}^{q+1}(\Sigma \iota)=(\Sigma \Delta)^{*}\left(\Sigma(f(\wedge f))^{*}(\Sigma(\iota \wedge \iota))\right. \\
& =\Sigma\left(\bar{\Delta}^{*}\left(f^{*} \iota \wedge f^{*} \iota\right)\right)=\Sigma \bar{\Delta}^{*}(x \wedge x)=\Sigma\left(x^{2}\right) .
\end{aligned}
$$

This completes the proof of Theorem (3.1).

As a corollary one gets the following result due to M. Barratt. Let $X_{n}=$ $S^{n} \cup_{a} e^{n+1}$ where $a: S^{n} \rightarrow S^{n}$ is the map of degree 2. Then $X_{n+1} \cong \Sigma X_{n}^{n}$ and thus $X_{n}$ is a suspension for $n>1$. Hence we may add maps.

Corollary (M. Barratt). 2(I) is not nullbomotopic where $I: X_{n} \rightarrow X_{n}$ is the identity, $n>1$.

Proof. Take $f: X_{1} \rightarrow K\left(Z_{2}, 1\right), f^{*} \iota=x$, the generator of $H^{1}\left(X_{1} ; Z_{2}\right)$. Since $x^{2} \neq 0$, by (3.1) $2 \Sigma f$ is detected by the functional $\mathrm{Sq}^{2}$, and hence $2 \Sigma f$ is not nullhomotopic. By suspension, $2\left(\Sigma^{n} f\right)$ is detected by $\mathrm{Sq}^{2}$ also and $2\left(\Sigma^{n} f\right)$ is also not nullhomotopic. But $2\left(\Sigma^{n} f\right)=\left(\Sigma^{n} f\right)(2(I))$ and hence $2(I)$ is not nullhomotopic.

Let $M^{2 q}$ be a manifold oriented in a cobordism theory with $v_{q+1}=0$, with $W^{2 q+k}$ a universal manifold for this theory, for dimension $2 q$. Let $g: W / \partial W \rightarrow$ $\Sigma^{k} M_{+}, c: M \rightarrow W$ be the spectral orientation and classifying maps respectively, so that $b=\left(\Sigma^{k} c\right) g: W / \partial W \rightarrow \Sigma^{k} W_{+}$is the spectral cobordism invariant of $M$ (see $\$ 1$ ).

(3.7) Lemma. Let $x \in H^{q}\left(M ; Z_{2}\right)$. Then in $2 M=M \cup M$,

$$
\psi(x \oplus x)=x^{2}[M]
$$

where $x \otimes x \in H^{q}\left(M ; Z_{2}\right)+H^{q}\left(M ; Z_{2}\right)=H^{q}\left(M \cup M ; Z_{2}\right)$. 
Proof. By (2.9), $g_{2}=g+g: W / \partial W \rightarrow \Sigma^{k} M_{+} \vee \Sigma^{k} M_{+}$is the spectral orientation map for $2 M$. Hence $g_{2}^{*} \Sigma^{k}(x \oplus x)=g^{*} \Sigma^{k} x+g^{*} \Sigma^{k} x=0$ so that $x \oplus x \in\left(\operatorname{ker} \eta^{*}\right)^{q}$, $\left(\eta^{*}=g^{*} \Sigma^{k}\right)$, and $\psi(x \oplus x)$ is defined.

Now if $\delta: M_{+} \rightarrow K\left(Z_{2}, q\right), \delta^{*} \iota=x$, then $\delta \cup \delta:(M \cup M)_{+} \rightarrow K\left(Z_{2}, q\right)$ represents $x \oplus x,(\delta \cup \delta)^{*} \iota=x \oplus x$, and

$$
\Sigma^{k}(\delta \cup \delta)=\Sigma^{k} \delta \vee \Sigma^{k} \delta: \Sigma^{k} M_{+} \vee \Sigma^{k} M_{+} \rightarrow \Sigma^{k} K\left(Z_{2}, q\right) .
$$

Hence $\left(\Sigma^{k}(\delta \cup \delta)\right) g_{2}$ is given by

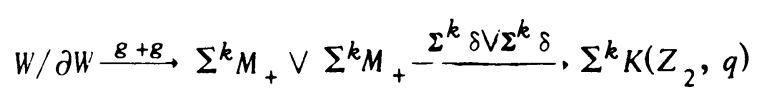

so that the composite is equal to $2\left(\left(\Sigma^{k} \delta\right) g\right)$. We may assume $k$ is very large so we are in the stable range, or we may suspend once to make $g$ a suspension map, so that $2\left(\left(\Sigma^{k} \delta\right) g\right)=\left(2 \Sigma^{k} \delta\right) g$. Then

$$
\begin{aligned}
\mathrm{Sq}_{2\left(\left(\Sigma^{k} \delta\right) g\right)}^{q+1}\left(\Sigma^{k} \iota\right) & =\operatorname{Sq}_{\left(2 \Sigma^{k} \delta\right) g}^{q+1}\left(\Sigma^{k} \iota\right)=g^{*} \operatorname{Sq}_{\left(2 \Sigma^{k} \delta\right)}^{q+1}\left(\Sigma^{k} \iota\right) \\
& =g^{*} \Sigma^{k}\left(\left(\delta^{*} \iota\right)^{2}\right)=g^{*} \Sigma^{k}\left(x^{2}\right), \quad \text { by (3.5) and (3.1). }
\end{aligned}
$$

Hence

$$
\begin{aligned}
\psi(x \oplus x) & =\left(\mathrm{Sq}_{2\left(\Sigma^{k} \delta\right) g}^{\mathrm{q}}\left(\Sigma^{k}()\right)[W]\right. \\
& =g^{*} \Sigma^{k}\left(x^{2}\right)[W]=\left(\Sigma^{k} x^{2}\right) g_{*}[W]=\left(\Sigma^{k} x^{2}\right)\left(\Sigma^{k}[M]\right)=x^{2}[M],
\end{aligned}
$$

(since $\left.g_{*}[W]=\left[M \times R^{k}\right]=\Sigma^{k}[M]\right)$. This completes the proof of (3.7).

(3.8) Theorem. $k(2 M)$ is defined if and only if $\left(c^{*} y\right)^{2}[M]=0$ for all $y \epsilon$ $H^{q}\left(W ; Z_{2}\right)$.

(Note that if $k(M)$ and $k(2 M)$ are defined then $k(2 M)=2 k(M)=0$ by (2.14); in fact $k(n M)=n k(M)$ by (2.13).)

Proof. By (2.9) if $c_{2}: M \cup M \rightarrow W$, then im $c_{2}^{*}=\left\{\left(c^{*}(y) \oplus c^{*}(y)\right), y \in H^{q}\left(W ; Z_{2}\right)\right\}$. By (2.6), $k(2 M)$ is defined if and only if $\psi\left(\operatorname{im} c_{2}^{*}\right)=0$, since $\eta_{2}^{*} c_{2}^{*}=b_{2}^{*}=(2 b)^{*}=$ $2 b^{*}=0$ by (2.9) so that im $c_{2}^{*} \subset \operatorname{ker} \eta_{2}^{*}$. By (3.7), $\psi\left(c^{*} y \oplus c^{*} y\right)=\left(c^{*} y\right)^{2}[M]$, and (3.8) follows.

Now we have the equivalent formulation:

(3.9) Corollary. $k(2 M)$ is defined if and only if, for $M, v_{q}(M) \in$ ker $\eta^{*}$, or in other words, $b^{*} v_{q}=0$. 
Proof. By (3.8), $k(2 M)$ is defined if and only if $\left(c^{*}(y)\right)^{2}[M]=\left(v_{q} \cup c^{*} y\right)[M]=0$ for all $y \in H^{q}\left(W ; Z_{2}\right)$, where $v_{q} \in H^{q}\left(M ; Z_{2}\right)$ is the qth Wu class (see $\$ 1$ ). In other words $k(2 M)$ is defined if and only if all characteristic numbers involving $v_{q}$ are zero. By (2.16), all characteristic numbers involving $v_{q}$ are zero if and only if $b^{*} v_{q}=0$. But $b^{*}=\eta^{*} c^{*}$, so $b^{*} v_{q}=0$ if and only if $\eta^{*}\left(c^{*} v_{q}\right)=\eta^{*}\left(v_{q}(M)\right)=0$. This proves (3.9).

If $k(2 M)$ is defined we would like to calculate its value. If $k(M)$ is defined it follows from (2.14) that $k(2 M)=2 k(M)$, so we must consider the case where $k(M)$ is not defined.

(3.10) Theorem. Suppose $k(M)$ is not defined, but that $k(2 M)$ is defined. Then $k(2 M)=\psi\left(v_{q}(M)\right)$.

(Note that by (3.9), $k(2 M)$ is defined implies that $v_{q}(M) \in \operatorname{ker} \eta^{*}$, so that $\psi\left(v_{q}(M)\right)$ is defined. $)$

(3.11) Corollary. $k\left(4 M^{2 q}\right)=\left(v_{q}(M)\right)^{2}[M]$.

(A similar result was obtained independently by E. H. Brown [25].)

Proof of Corollary. The spectral cobordism invariant $b_{4}$ of $4 M$ is given by $b_{4}=4 b$, by (2.9), so that $\operatorname{ker}(2 b)^{*}=H^{*}\left(\Sigma^{k} W_{+} ; Z_{2}\right)$ and $\mathrm{Sq}_{4 b}^{q+1}=2 \mathrm{Sq}_{2 b}^{q+1}=0$, so so that $k(4 M)$ is defined. If $k(2 M)$ is defined, then by (3.9) $v_{q}(M) \in \operatorname{ker} \eta^{*} \cap$ im $c^{*}$ so that $\left(v_{q}(M)\right)^{2}[M]=0$, since im $c^{*}$ annihilates $\operatorname{ker} \eta^{*^{q}}$ by (2.2). But by (2.14), $k(4 M)=2(k(2 M))=0$ if $k(2 M)$ is defined.

If $k(2 M)$ is not defined, then $k(4 M)=\psi\left(v_{q}(2 M)\right)$ by $(3.10)$ and $v_{q}(2 M)=$ $v_{q}(M) \oplus v_{q}(M) \in H^{q}\left(M ; Z_{2}\right)+H^{q}\left(M ; Z_{2}\right)$. Hence by (3.7), $\psi\left(v_{q}(2 M)\right)=$ $\psi\left(v_{q}(M) \oplus v_{q}(M)\right)=\left(v_{q}(M)\right)^{2}[M]$ and $k(4 M)=\psi\left(v_{q}(2 M)\right)$ by (3.10), which proves (3.11).

Proof of (3.10). We suppose that $k(M)$ is not defined but $k(2 M)$ is defined, so that $v_{q}(M) \in\left(\operatorname{ker} \eta^{*}\right)^{q}$ by (3.9). Let $R=\left(\operatorname{ker} \eta^{*} \cap \operatorname{im} c^{*}\right)^{q}=$ radical of $($,$) on$ ker $\eta^{*^{q}}$, and note that $\psi \mid R$ is linear, $\psi \mid R: R \rightarrow Z_{2}$. Let $R^{\prime}=\operatorname{ker}(\psi \mid R)$, so that $R^{\prime}$ is of codimension 1 in $R$. Choose a complementary subspace $N$ to $R$ in ker $\eta^{*}$, so that $($,$) is nonsingular on N$, and choose a complementary subspace $A$ to ker $\eta^{*}$ in $H^{q}\left(M ; Z_{2}\right)$ such that $A$ is orthogonal to $N$ (i.e. let $A C$ annihilator of $N$ in $\left.H^{q}\left(M ; Z_{2}\right)\right)$. Then $H^{q}\left(M ; Z_{2}\right)=N+R+A$, and since $($,$) is non-$ singular on $N$, it is nonsingular on $R+A$ and these two subspaces are orthogonal. Since $A \cap \operatorname{ker} \eta^{*}=0, \eta^{*} \mid A$ is injective.

Then in $2 M, H^{q}\left(2 M ; Z_{2}\right)=H^{q}\left(M ; Z_{2}\right)+H^{q}\left(M ; Z_{2}\right)$ so $H^{q}\left(2 M ; Z_{2}\right)=N+N+$ $R+R+A+A$. Now $\eta_{2}^{*}: H^{q}\left(2 M ; Z_{2}\right) \rightarrow H^{q+k}\left(W / \partial W ; Z_{2}\right)$ is given by $\eta_{2}^{*}=\eta^{*}+\eta^{*}$, by (2.9). Hence ker $\eta_{2}^{*}=\operatorname{ker} \eta^{*}+\operatorname{ker} \eta^{*}+\Delta A$ where $\Delta: H^{q}\left(M ; Z_{2}\right) \rightarrow H^{q}\left(M ; Z_{2}\right)+H^{q}\left(M ; Z_{2}\right)$ is given by $\Delta x=x \oplus x$. Similarly 
im $c_{2}^{*}=\Delta\left(\right.$ im $\left.c^{*}\right)$; and since $\eta_{2}^{*} c_{2}^{*}=b_{2}^{*}=2 b^{*}=0$, im $c_{2}^{*} c \operatorname{ker} \eta_{2}^{*}$ and im $c_{2}^{*}=$ radical of (, ) on $\operatorname{ker} \eta_{2}^{*}$, by (2.2). Hence ker $\eta_{2}^{*} / \operatorname{im} c_{2}^{*} \cong N_{1}+N_{2}+R+\bar{A}$ where $\bar{A}=\Delta A /$ im $c_{2}^{*} \cap \Delta A$, since $R C$ im $c^{*}$, identifying $R+R / \Delta R$ with $R$. It follows that the two copies of $N$ and $(R+\bar{A})$ are orthogonal under $($,$) and ($, is nonsingular on each term. Hence if $\psi_{2}: \operatorname{ker} \eta_{2}^{*} \rightarrow Z_{2}$ is the quadratic form, $c\left(\psi_{2}\right)=c\left(\psi_{2} \mid N_{1}\right)+c\left(\psi_{2} \mid N_{2}\right)+c\left(\psi_{2} \mid R+\bar{A}\right)$. By (2.10), $\psi_{2}\left|N_{i}=\psi\right| N$, so that $c\left(\psi_{2} \mid N_{i}\right)=c(\psi \mid N), i=1,2$, so that $c\left(\psi_{2}\right)=2 c(\psi \mid N)+c\left(\psi_{2} \mid R+\bar{A}\right)=$ $c\left(\psi_{2} \mid R+\bar{A}\right)$.

Now $R=$ radical of $\psi$ on ker $\eta^{*}$ is a self-annihilating subspace of ker $\eta_{2}^{*} /$ im $c_{2}^{*}$, and if $a \oplus a, b \oplus b \in \Delta A$, then $(a \oplus a, b \oplus b)=(a, b)+(a, b)=0$ so $\Delta A$ and hence $\bar{A}$ are self-annihilating subspaces of $\operatorname{ker} \eta_{2}^{*}$ and $\operatorname{ker} \eta_{2}^{*} / \operatorname{im} c_{2}^{*}$ respectively. Since (, ) is nonsingular on $R+\bar{A}$, it follows that $R$ and $\bar{A}$ are dually paired by (, ), and hence any basis for $R$ is half of a symplectic basis for $R+\bar{A}$. Since $\psi\left|R=\psi_{2}\right| R$ by (2.10), and $\psi \mid R^{\prime} \equiv 0$, it follows that if $\bar{a} \in \bar{A}$ such that, for $r^{\prime} \in R^{\prime},\left(r^{\prime}, \bar{a}\right)=0$, then a basis for $R^{\prime}$ together with $x \in R$ such that $(x, \bar{a})=1$ are half of a sympletic basis for $\operatorname{ker} \eta_{2}^{*} / \operatorname{im} c_{2}^{*}$ and $c\left(\psi_{2}\right)=$ $\psi_{2}(x) \psi_{2}(\bar{a})=\psi_{2}(\bar{a})$, since $x \notin R^{\prime}=\operatorname{ker}(\psi \mid R)$.

Now $\bar{a} \in \bar{A}$ is represented by $a \oplus a \in \Delta A$ and by (3.7), $\psi_{2}(a \oplus a)=a^{2}[M]$, $a \in H^{q}\left(M ; Z_{2}\right)$. But $a^{2}[M]=\left(a v_{q}(M)\right)[M]$, so that $\psi_{2}(\bar{a})=\left(a v_{q}(M)\right)[M]$. Now $\bar{a}$ orthogonal to $R^{\prime}$ is the same as $a$ orthogonal to $R^{\prime}$, so that a generates the annihilator of $R^{\prime}$ in $A$. Hence $\left(a v_{q}(M)\right)[M]=\left(a, v_{q}(M)\right)=0$ if and only if

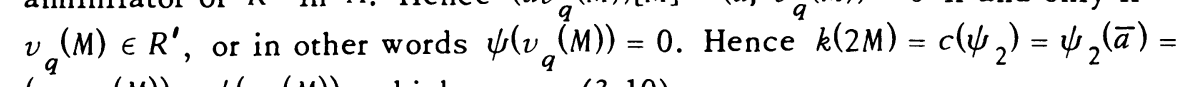
$\left(a, v_{q}(M)\right)=\psi\left(v_{q}(M)\right)$, which proves (3.10).

Finally combining all the results of this section we get the following formula which will be useful in $\$ 4$.

(3.12) Corollary. Let $M^{2 q}$ be such that (a) $k(M)$ is defined, and (b) $\left(v_{q}(M)\right)^{2}[M] \neq 0$. Then for any integer $n, k(n M)$ is defined if and only if $n \neq$ $2(\bmod 4)$ and

$$
\begin{aligned}
k(n M) & =k(M) & & \text { for } n \equiv \pm 1 \quad(\bmod 8), \\
& =k(M)+1 & & \text { for } n \equiv \pm 3 \quad(\bmod 8), \\
& =1 & & \text { if } n \equiv 4(\bmod 8), \\
& =0 & & \text { if } n=n \quad(\bmod 8)
\end{aligned}
$$

The typical example of such $M$ we use in $\$ 4$ is projective space $P^{2 q}$, or some $M$ with a normal map into $P^{2 q}$.

Proof. Since $\eta^{*}\left(v_{q}(M)\right)=\eta^{*} c^{*} v_{q}=b^{*} v_{q} \neq 0$ and since $\left(v_{q}(M)\right)^{2}[M] \neq 0$ (cf. (2.16)), it follows from (3.9) that $k(2 M)$ is not defined. For $s M, b_{s}=s b$; so for 
$s$ odd, $b_{s}^{*}=s b^{*}=b^{*}$, and by (3.9) $k(2 s M)$ is not defined for $s$ odd. Since $b_{2}^{*}=$ $2 b^{*}=0$, it follows from (3.9) that $k(4 M)$ and $k(8 M)$ are defined, so by (3.11), $k(4 M)=1$, and by (2.14) or (2.15), $k(8 M)=0$. Since $b_{4}^{*}=4 b^{*}=0$ and $k(4 M)$ is defined it follows from (2.13) that $k(5 M)$ is defined and $k(5 M)=k(M)+k(4 M)=$ $k(M)+1$. Now $k(-M)$ is defined if $k(M)$ is, and $k(-M)=k(M)$ (cf. (3.5)). Hence, by (2.13), $k(3 M)$ is defined and $k(3 M)=k(-M)+k(4 M)=k(M)+1$. It follows from (2.13) that $k(7 M)$ is defined and $k(7 M)=k(8 M)+k(-M)=k(M)$, and periodicity mod 8 follows also from (2.13).

4. Applications to surgery and fixed point free involutions. Let $M^{2 q}$ be a smooth (or piecewise linear) manifold, and let $\xi^{k}, k \gg 2 q$, be a linear (or p.1.) bundle over $M^{2 q}$. A normal map $(f, b)$ (see $[8, \S 2]$ ) consists of a map of degree 1 , $f: M^{\prime} \rightarrow M, M^{\prime}$ a smooth (or p.l.) $2 q$-manifold, $b: \nu^{k} \rightarrow \xi^{k}$ a linear (or p.l.) bundle map covering $f, \nu^{k}=$ normal bundle of $M^{\prime}$ in $S^{2 q+k}$. We will use $M$ as the classifying space, and the total space of $E\left(\xi^{-1}+\tau^{-1}\right)=W, \tau=$ tangent bundle of $M$, as the universal manifold to define the spectral orientations and the Kervaire invariant. The existence of the normal map $(f, b)$ ensures that $v_{q+1}(\xi)=0$ so that $W$ can be used to define the Kervaire invariant (see [8, Chapter 3]). In fact we have the following:

(4.1) Theorem. With $(f, b)$ defining the orientation, $k\left(M^{\prime}\right)$ is defined and if $q$ is odd and $M$ is 1-connected or if $M$ is nonorientable and $\pi_{1}(M)=Z_{2}$, then $k\left(M^{\prime}\right)$ is the obstruction to surgery, i.e., the obstruction to finding a normal cobordism of $(f, b)$ to a bomotopy equivalence.

This is proved in $\left[8, \S_{3}\right]$ for $q$ odd, $M$ oriented (no other hypothesis on $M$ ). If $M$ is nonorientable, then Wall [29] has shown the obstruction to surgery is the Arf invariant of a quadratic form from $H^{q}\left(M^{\prime} ; Z_{2}\right)$ to $Z_{2}$. Using this, the same proof as in $[8, \$ 3]$ holds.

Now we will use the results of $\$ 3$ to construct normal maps with surgery obstruction $k\left(M^{\prime}\right) \neq 0$ for particular kinds of manifolds $M$.

Let $N^{m}$ be a smooth (or p.l.) m-manifold and $T: N^{m} \rightarrow N^{m}$ a smooth (or p.l.) fixed point free involution. Let $M^{m}=N / T$ be the quotient manifold, $\xi^{k}$ the normal bundle of $M^{m}$ in $S^{m+k}$, and let $(f, b)$ be a normal map $f: M^{\prime} \rightarrow M, b: \nu \rightarrow \xi$, as above.

We shall define the operation of multiplication by an odd integer on normal maps as follows:

(4.2) Definition. Let $n=2 s+1$, and define $n(f, b)=(g, c), g: M^{\prime}(n) \rightarrow M$, $M^{\prime}(n)=n M^{\prime} \cup-s N$ where $n M^{\prime}=M^{\prime} \cup \ldots \cup M^{\prime}, n$ times, $-s N=(-N) \cup \cdots \cup(-N)$, $s$ times, $-N$ is $N$ with the opposite orientation. Then $g=f$ on each copy of $M^{\prime}$, $g=2$-fold covering map $p$ on each copy of $N$, so 


$$
\text { degree } g=n \text { degree } f-s \text { degree } p=n-2 s=1 \text {. }
$$

Then $c=b$ over each $M$, and a fixed map $d$ covering $p$ over $N$, where $d$ is the inverse of $d p: \tau_{N} \rightarrow \tau_{M}$. Hence $(g, c)$ is a normal map.

We will be interested in computing the invariants of $n(f, b)$ in terms of the invariants of $(f, b)$. We will start with the spectral orientation map and spectral cobordism invariant.

Since $\xi^{k}$ is the normal bundle of $M$, it follows that $W=M \times R^{k}$ is the universal manifold. Then the map $d p: \tau_{N} \rightarrow \tau_{M}$ stably is the differential of an embedding $e: N \times R^{k} \rightarrow M \times R^{k}$ unique up to isotopy, using the Hirsch immersion theorem and general position. Then the embedding of $M^{\prime}(n) \times R^{k} \subset M \times R^{k}$ is the disjoint union of $M^{\prime} \times R^{k} \subset M \times R^{k}$ corresponding to $(f, b)$, and $e: N \times R^{k} \subset M \times R^{k}$, translated by some values in $R^{k}$ to make them disjoint. Hence it follows:

(4.3) Proposition. The spectral orientation map corresponding to $n(f, b)$ is the composite

$$
\Sigma^{k} M_{+} \stackrel{\delta}{\longrightarrow} \bigvee_{n+s} \Sigma^{k} M_{+} \stackrel{\stackrel{V_{g} \bigvee \bigvee_{s}^{r}}{\longrightarrow}}{\longrightarrow} \bigvee_{n} \Sigma^{k} M_{+}^{\prime} \vee \bigvee_{s} \Sigma^{k} N_{+}
$$

where $\delta$ is the map which pinches $n+s-1$ copies of $\Sigma^{k-1} M_{+}$to a point, $g$ is the spectral orientation of $M^{\prime}$ given by $(f, b)$, and $r$ is the spectral orientation of $N$ given by $d$.

Now the classifying map of $M^{\prime}(n)$ is $f$ on each copy of $M^{\prime}$ and $p$ on each copy of $N$ so that the spectral cobordism invariant is the composite

$$
\Sigma^{k} M_{+} \stackrel{\delta}{\rightarrow} \bigvee_{n+s} \Sigma^{k} M_{+} \underline{\bigvee g \bigvee V r} \rightarrow \underset{n}{\bigvee} \Sigma^{k} M_{+}^{\prime} \vee \bigvee_{s} \Sigma^{k} N_{+} \stackrel{\bigvee \rho \vee \bigvee p}{\longrightarrow} \Sigma^{k} M_{+}
$$

and hence we get

(4.4) Proposition. The spectral cobordism invariant of $n(f, b), n=2 s+1$, is given by

$$
b\left(M^{\prime}(n)\right)=n b\left(M^{\prime}\right)-s b(N)
$$

where $h()$ denotes the spectral cobordism invariant of the corresponding manifold and the suspension structure of $\Sigma^{k} M_{+}$defines the sum.

Since $n=2 s+1$ we can also rewrite (4.4) as

$$
b\left(M^{\prime}(n)\right)=b(M)+s(2 b(M)-b(N))
$$

which will be convenient later. 
Now let suppose $m=2 q$ and let us consider the following additional assumption:

(4.6) If $b_{N}$ is the spectral cobordism invariant of $N$, assume

(a) $b_{N}^{*}: H^{q+k}\left(\Sigma^{k} M_{+} ; Z_{2}\right) \rightarrow H^{q+k}\left(\Sigma^{k} M_{+} ; Z_{2}\right)$ is the zero map;

(b) $k(N)$ is defined and $k(N)=0$ (with the orientation induced by $p$ and $d$ ).

(4.7) Lemma. Under the assumption (4.6), if $n$ is odd, $k\left(M^{\prime}(n)\right)$ is defined and $k\left(M^{\prime}(n)\right)=k\left(n M^{\prime}\right)$.

This follows immediately from (2.13) and the fact that $k\left(n M^{\prime}\right)$ is defined since $n M^{\prime} \rightarrow M$ is of degree $1 \bmod 2$ if $n$ is odd.

(4.8) Theorem. Suppose $M, N,(f, b)$ etc. as above and suppose (4.6) is satisfied and $\left(\nu_{q}(M)\right)^{2}[M] \neq 0$. Then

$$
\begin{aligned}
k\left(M^{\prime}(n)\right) & =k\left(M^{\prime}\right) & & \text { for } n \equiv \pm 1 \quad(8), \\
& =k\left(M^{\prime}\right)+1 & & \text { for } n \equiv \pm 3(8) .
\end{aligned}
$$

Proof. Since $(f, b), f: M^{i} \rightarrow M$ is a normal map, $f$ is of degree 1 , so $v_{q}\left(M^{\prime}\right)=$ $f^{*}\left(v_{q}(M)\right), f_{*}\left[M^{\prime}\right]=[M]$, and $\left(v_{q}\left(M^{\prime}\right)\right)^{2}\left[M^{\prime}\right]=\left(f^{*}\left(v_{q}(M)\right)^{2}\right)\left[M^{\prime}\right]=\left(v_{q}(M)\right)^{2} f_{*}\left[M^{q^{\prime}}\right]=$ $\left(v_{q}(M)\right)^{2}[M] \neq 0$. Then (4.8) follows from (4.7) and (3.12).

The obvious situation to apply these results is where $M=P^{m}$, the real projective space, so $N=S^{m}$.

(4.9) Corollary. For any bomotopy real projective space $M^{2 q}$, there are normal maps ( $n$ (identity), $n \equiv \pm 3(8)$ ) of manifolds into $M^{2 q}$ (with its normal bundle) of Kervaire invariant (i.e. the surgery obstruction) nonzero.

Proof. Simply take $(f, b)=$ identity, and apply (4.8). It follows that for $n \equiv$ $\pm 3(\bmod 8), n(f, b)$ has Kervaire invariant 1 .

(4.10) Corollary. There are smooth normal maps into real projective space $P^{2 q}$ with Kervaire invariant 1 (i.e. $n$ (identity) for $n \equiv \pm 3(8)$ ).

This was known in the p.l. case by S. Lopez de Medrano [18] and Wall [30].

Let $f: M^{m} \rightarrow P^{m}$ be a homotopy equivalence. Taking $\xi^{k}$ over $P^{m}$ to be $f^{-1 *}(\nu M)$, we may define a normal map $(f, b)$ into $P^{m}, \xi^{k}$. Then if we make $f$ transverse regular on $P^{2 q} \subset P^{m}, 2 q<m$, we get a normal map $\left(f_{q^{\prime}} b_{q}\right): f_{q}$ : $f^{-1}\left(P^{2 q}\right) \rightarrow P^{2 q}, b_{q}$ induced by $b$ and $f \mid$ tubular neighborhood of $f^{-1}\left(P^{2 q}\right)$. Let us define

(4.11) Definition. $k_{q}(M)=k\left(f^{-1}\left(P^{2 q}\right)\right) \in Z_{2}$, i.e., $k_{q}(M)=$ Kervaire invariant of $f^{-1}\left(P^{2 q}\right)$ using the normal map $\left(f_{q}, b_{q}\right)$ to define the orientation (as at the beginning of $\S 4)$. 
(4.12) Proposition. $k_{q}(M)$ depends only on the piecewise linear equivalence class of $M$, in particular, it is independent of the choice of the bomotopy equivalence $f$ or the bundle map $b$.

Proof. There is only one other homotopy equivalence $f^{\prime}: M \rightarrow P^{2 q}$ up to homotopy, i.e., the equivalence of opposite degree from $f$. But clearly $f^{\prime}$ with any choice of bundle map $b^{\prime}$ is normally cobordant to $\left(f \cup p, b^{\prime \prime}\right), f \cup p: M \cup\left(-S^{m}\right) \rightarrow$ $P^{m}, p: S^{m} \rightarrow P^{m}$ the twofold cover. Hence $f^{\prime-1}\left(P^{2 q}\right)$ is normally cobordant (using transversality) to $f_{q} \cup p_{q}: f^{-1}\left(P^{2 q}\right) \cup\left(-S^{2 q}\right) \rightarrow P^{2 q}$ so by Lemma 4.7, the Kervaire invariant defined by $\left(f^{\prime}, b^{\prime}\right)$ is the same as that defined by $\left(f, b^{\prime \prime}\right)$. It remains to show it is independent of the choice of bundle map.

If $b, b^{\prime}: \nu \rightarrow \xi$ are two bundle maps covering the homotopy equivalence $f: M^{m} \rightarrow P^{m}$, then there is a bundle map $c: \xi \rightarrow \xi$ such that $b^{\prime}=c b$. Therefore, $b_{q}^{\prime}=c_{q} b_{q}$, where subscript $q$ denotes restriction to $f^{-1}\left(P^{2 q}\right)$ or to $P^{2 q}$. It follows that the spectral orientation maps corresponding to $b_{q}$ and $b_{q}^{\prime}$ differ by the homotopy equivalence of $\Sigma^{k} P^{2 q}$ which is $S$-dual to $T(c): T(\xi) \rightarrow T(\xi)$ so that the quadratic forms and the value of $k\left(f^{-1}\left(P^{2 q}\right)\right)$ are unchanged.

Clearly if $g: M_{1} \rightarrow M_{2}$ is a p.l. equivalence, and if $f_{1}=g_{2} g$, then $g^{-1} f_{2}^{-1}\left(P^{2 q}\right)=f_{1}^{-1}\left(P^{2 q}\right)$ and it is clear that the Kervaire invariants are the same. This completes the proof of (4.12).

(4.13) Proposition. If $m=4 q+1$, then $k_{2 q}(M)=$ desuspension invariant de. fined in [10].

This result has been proved by many people, e.g. Wall [30], Lopez de Medrano [18], and Berstein [5].

(4.14) Proposition. $k_{2 q-1}(M)=k_{2 q}(M)$, all $q$.

This has also been proved by many people including Lopez de Medrano [18], Wall [30], Orlik and Rourke and others.

One may ask the question, what sequences $k_{2}, k_{4}, \ldots, k_{2 q} \in Z_{2}$ actually arise as the Kervaire invariants $k_{2 i}(M)$ for a homotopy projective space $M$. Lopez de Medrano [18] and Wall [30] have shown that for piecewise linear $M$, all such sequences can occur. We shall study this question in the smooth case.

(4.15) Theorem. Suppose a sequence $K=\left\{k_{2}, k_{4}, \ldots, k_{2 q}\right\}$ occurs as $k_{2 i}=$ $k_{2 i}\left(M^{m}\right), m=4 q+1, M^{m}$ a smooth (or p.l.) bomotopy real projective space. Then the sequence $K^{\prime}=\left\{K_{2}^{\prime}, \ldots, k_{2 q}^{\prime}\right\}, k_{2 i}^{\prime}=k_{2 i}+1$, also occurs for some smooth $M^{\prime}$ (or p.l. $M^{\prime}$ with the same stable normal bundle as $M$ ).

Proof. Let $M^{\prime \prime}=M(n), n$ odd, as in (4.2). Then $(n f): M(n) \rightarrow P^{m}$, and it follows easily from the definition (4.2) that $(n f)^{-1}\left(P^{2 i}\right)=\left(f^{-1}\left(P^{2 i}\right)\right)(n)$. Now it follows from results of Wall [29] that in dimension $m=4 q+1$ any normal map into 
$P^{m}$ is normally cobordant to a homotopy equivalence, so that $M^{\prime \prime}$ is normally cobordant to $M^{\prime}, f^{\prime}: M^{\prime} \rightarrow P^{m}$ a homotopy equivalence. Using transversality, it follows that $f^{\prime-1}\left(P^{2 i}\right)$ is normally cobordant to $(n f)^{-1}\left(P^{2 i}\right)$, so that $k_{2 i}\left(M^{\prime}\right)=$ $k\left(\left(f^{-1}\left(P^{2} i\right)\right)(n)\right)=k_{2 i}(M)+1$, if $n \equiv \pm 3(\bmod 8)$ by $(4.8)$, which completes the proof of (4.15).

Taking $K=(0, \ldots, 0)$ (with $\left.M=P^{m}\right)$ we deduce from (4.15)

(4.16) Corollary. There are smooth homotopy projective $(4 \mathrm{q}+1)$ spaces $M$ (normally cobordant to $P^{4 q+1}(n)$ for $n \equiv \pm 3(8)$ ) such that $k_{2 i}(M)=1$, all $i$, so that in particular the desuspension invariant is nonzero.

Different constructions leading to a similar result have been found independently by Berstein [5] and Giffen [13]. .Giffen's examples are on the Brieskorn spheres, a result which we also found (see $\$ 6$ ).

If we define $f^{-1}\left(P^{t}\right) \subset M, f: M \rightarrow P^{m}$, as a $t$ dimensional characteristic submanifold of $M$, one may ask for what $t$ a given $M$ admits a characteristic submanifold $Q^{t} \subset M$ which is a homotopy projective space. This is equivalent to an analogous question about "characteristic" homotopy spheres in dimension $t$ in the 2-fold covering space $p: N \rightarrow M$. We remark that one may show, using the results of Wall [30], Lopez de Medrano [18], and Browder [9]:

(4.17) Proposition. The manifold $M$ of (4.16) admits homotopy projective spaces as characteristic submanifolds only in dimensions $t \equiv 1(\bmod 4)$.

We can use our technique to study the twofold cover $p: N \rightarrow M$.

(4.18) Proposition. For $M$ the manifold of (4.16), the twofold cover $N$ represents a generator of $b P^{4 q+2}$, i.e. it is the Kervaire sphere which bounds a framed manifold of Kervaire invariant 1.

Proof. By the construction, $M$ is normally cobordant to $P^{m}(n), n \equiv \pm 3$ $(\bmod 8)$. Now $P^{m}(n) \subset P^{m+1}(n)$ and it follows easily that $M^{m} \subset W^{m+1}, W^{m+1}$ normally cobordant to $P^{m+1}(n)$. For if $\partial U=M \cup P^{m}(n), g: U \rightarrow P^{m}$, then take $E\left(g^{*}(\alpha)\right)=$ the total space of the line bundle $g^{k}(\alpha), \alpha=$ canonical line bundle over $P^{m}$, and take the union $V^{m+2}=E\left(g^{*}(\alpha)\right) \cup\left(P^{m+1}(n) \times I\right)$ with $E\left(\left(g \mid P^{m}(n)\right)^{*}(\alpha)\right)$ identified with the normal bundle of $P^{m}(n) \subset P^{m+1}(n) \times 0$. Then $\partial V=$ $W \cup P^{m+1}(n) \times 1$ and $M^{m} \subset W^{m+1}$ as required (compare the construction in [9]).

Now the normal bundle of $M^{m} C W^{m+1}$ is $(g \mid M)^{*}(\alpha)$ which is the canonical line bundle over $M$ so that the boundary is $N^{m}$ the 2-fold cover of $M^{m}$. By (4.8), $P^{m+1}(n)$ has Kervaire invariant 1 if $n \equiv \pm 3(\bmod 8)$, so that $k(W)=1$. But $f$ : $W \rightarrow P^{m+1}$ with $M=f^{-1}\left(P^{m}\right), f \mid M$ a homotopy equivalence, so that it follows that $k(W)=k\left(W-E\left(a^{\prime}\right)\right)=1$ where $a^{\prime}=$ normal bundle of $M \subset W$, and $\partial\left(W-E\left(\alpha^{\prime}\right)\right)=N$ (compare [8, (III.4.14)]). But $f: W-E\left(\alpha^{\prime}\right) \rightarrow P^{m+1}-E(\alpha)=D^{m+1}$, 
a disk, $f \mid N: N^{m} \rightarrow S^{m}$ a homotopy equivalence, so that it follows that $W-E\left(\alpha^{\prime}\right)$ is a framed manifold of Kervaire invariant 1 whose boundary is $N$. This proves (4.18).

In $\$ 5$ we shall show that the $N$ discussed above is a certain $(4 q+1)$ dimensional Brieskorn sphere so that (4.18) gives a new proof of the theorem due to Levine and Milnor $[20, \S 8]$ on the differential structure of these spheres.

The examples of (4.16) give smooth examples with homogeneous Kervaire invariants, i.e. with $k_{2 i}=k_{2 j}$, all $i, j<m / 2$. We conclude this section by constructing some inhomogeneous examples.

In his thesis, Sullivan [27] shows that there is a smooth $A^{8}$ homotopy equivalent to $C P^{4}$ with no characteristic 6-dimensional homotopy complex projective space, and which has $C P^{2}$ as a characteristic submanifold. An $S^{1}$-bundle $B^{9}$ over $A^{8}$ is then a homotopy $P^{9}$ with $P^{5}$ as a characteristic submanifold, so that $k_{2}\left(B^{9}\right)=0$. However, Montgomery and Yang [21] have shown that under these circumstances $B^{9}$ has a characteristic homotopy $P^{8}$ if and only if $A^{8}$ has a characteristic homotopy $C P^{3}$. Hence $k_{8}\left(B^{9}\right)=1$, since $k_{8}$ is the obstruction to finding such a characteristic homotopy $P^{8}$ (see (4.13)). Hence $K=\{0,1\}$ can be realized. Using the construction of (4.15), it follows that $K^{\prime}=\{1,0\}$ also occurs so that we get:

(4.19) Proposition. For smooth 9-dimensional bomotopy projective spaces all combinations $K=\left\{k_{2}, k_{4}\right\}$ can occur as Kervaire invariants.

5. Smooth invariants of fixed point free involutions. In this section we apply the results of $\$ \$ 1-4$ to study free involutions on homotopy spheres. Recall that if $T: \Sigma^{m} \rightarrow \Sigma^{m}$ is a fixed point free involution of a homotopy $m$-sphere $\Sigma$, then the orbit space $\Sigma / T$ is homotopy equivalent to real projective $m$-space $P^{m}$.

(5.1) Proposition. Let $M^{n}$ bave the bomotopy type of $P^{n}$. Then there are exactly two bomotopy classes of bomotopy equivalences of $M^{n}$ with $P^{n}$ determined by the degree (or the degree of the map of universal covering spaces $\left.N^{n} \rightarrow S^{n}\right)$.

For a proof we refer to Olum [23].

(5.2) Lemma. Suppose $M^{n}$ is a smooth homotopy real projective m-space, and let $f: M^{m} \rightarrow P^{m}$ be a bomotopy equivalence. Then $f^{*}\left(\nu_{P m}\right)=\nu_{M^{m}}$, where $\nu_{M}, \nu_{P}$ are the stable normal bundles in $S^{m+k}, k$ large.

Proof. From the result of Atiyah [2] and Hirsch [16], we know that $f^{*}\left(\nu_{P}\right)$ is fibre homotopy equivalent to $\nu_{M}$. If $J: K O() \rightarrow J()$ is the functor which sends a stable linear bundle into its fibre homotopy type, Adams [1] has shown that $J: K O\left(P^{m}\right) \rightarrow J\left(P^{m}\right)$ is an isomorphism, so that $f^{*}\left(\nu_{P}\right)$ and $\nu_{M}$ are linearly equivalent, proving (5.2). 
It follows from (5.2) that any smooth homotopy projective $m$-space $M^{m}$ admits normal maps into $P^{m}$, so that $M^{m}$ can be oriented in the theory with universal manifold $P^{m} \times R^{k}, k$ large, or $X^{m} \times R^{k}$ for any homotopy projective space $X$. We shall study such $M^{m}$ by means of this, through the spectral cobordism invariant of (1.4) and the Kervaire invariants of $\$ 4$.

Let $M^{m}$ be a homotopy real projective $m$-space, $N^{m} \rightarrow M^{m}$ its twofold covering, so that $N^{m}$ is a homotopy $m$-sphere, and choose an orientation of $N^{m}$. We will call this an oriented homotopy projective space. By (5.1) there is a unique (up to homotopy) homotopy equivalence $b: M^{m} \rightarrow P^{m}$ such that $\tilde{b}: N^{m} \rightarrow S^{m}$ is of degree 1. By (5.2), $b^{*}\left(\nu_{P m}\right)=\nu_{M}$, so that it follows that $b^{*}\left(\left[\tau_{P m}\right]\right)=\left[\tau_{M^{m}}\right]$ (where [ ] denotes the stable bundle). Hence, if we replace the composition $M \rightarrow$ $P^{m} \subset P^{m} \times R^{k}, k \gg m$, by an embedding, it follows that the normal bundle $\eta^{k}$ of $M^{m} \subset P^{m} \times R^{k}$ satisfies $[\eta]=f^{*}\left[\tau_{P m}\right]-\left[\tau_{M^{m}}\right]=0$, and since $k \gg m, \eta$ is the trivial bundle. Thus $M^{m} \times R^{k}$ is embedded in $P^{m} \times R^{k}$ as a tubular neighborhood of the embedded $M^{m}$. Since $k \gg m$ the homotopy class of $b$ determines the embedding of $M^{m}$ up to isotopy in $P^{m} \times R^{k}$, and by the tubular neighborhood theorem, another embedding of $M^{m} \times R^{k}$ as a tubular neighborhood of $M^{m}$ differs from the given one by a linear map on each fibre, or in other words by twisting each fibre by a map $a_{:} M^{m} \rightarrow O(k)$ (normalizing to make it orthogonal). If we orient $N^{m} \times R^{k}$ and $S^{m} \times R^{k}$ and require the induced embedding $N^{m} \times R^{k} \subset S^{m} \times R^{k}$ to be orientation preserving, then $\alpha: M^{m} \rightarrow S O(k)$. Further changing a by a homotopy changes the embedding by an isotopy, so that up to isotopy our only ambiguity is $\{a\} \in\left[M^{m}, S O(k)\right]=K O^{-1}(M)$ (since $k \gg m$ ).

(5.3) Proposition. $K O^{-1}\left(P^{m}\right)=Z_{2}$ for $m \not \equiv-1(\bmod 4), K O^{-1}\left(P^{m}\right)=Z_{2}+Z$ if $m \equiv-1(\bmod 4)$, where the $Z$ is the image of $K O^{-1}\left(S^{m}\right)$ under the map $j$ : $P^{m} \rightarrow S^{m}$ of degree 1 .

This is proved in [12].

In [26, Chapter IV] a map $\beta: P^{m} \rightarrow S O(m+1)$ is described with the property that $\beta^{*} \sigma^{*} w_{i+1}=x^{i}$, where $w_{i+1} \in H^{i+1}\left(B_{0} ; Z_{2}\right)$ is the $(i+1)_{\text {st }}$ Stiefel-Whitney class, $x \in H^{1}\left(P^{m} ; Z_{2}\right)$ is the generator, $\sigma^{*}: H^{i+1}\left(B_{0} ; Z_{2}\right) \rightarrow H^{i}\left(O ; Z_{2}\right)$ is the cohomology suspension. Hence the Stiefel-Whitney classes $w_{2}, \ldots, w_{m+1}$ of the corresponding bundle over $\Sigma P^{m}$ are nonzero and therefore the bundle is not fibre homotopically trivial. Hence we get

(5.4) Lemma. The class $\{\beta\} \in K O^{-1}\left(P^{m}\right)$ generates $Z_{2} \subset K O^{-1}\left(P^{m}\right)$, $J$ is a monomorphism on the $Z_{2}$ and the inclusion $j: P^{i} \subset P^{m}$ induces $j^{*}: K O^{-1}\left(P^{m}\right) \rightarrow$ $K O^{-1}\left(P^{i}\right)$ which is an isomorphism on $Z_{2}$ and annibilates the $Z$ factor.

Thus for a given homotopy projective space $M^{n}$ and an orientation of its twofold cover $N^{n}$, if $n \not \equiv-1(4)$, there are exactly two choices of normal maps 
$M^{n} \rightarrow X^{n}$ (or $X^{n} \times R^{k}$ orientations of $M^{n}$ ) where $X^{n}$ is a fixed homotopy projective space, corresponding to the two elements of $K O^{-1}\left(P^{n}\right)$, while for $n \equiv-1$ (4) there are other choices coming from the image of $K O^{-1}\left(S^{n}\right)$ in $K O^{-1}\left(P^{n}\right)$. If we take the spectral cobordism invariants in $\left[\Sigma^{k} X_{+}^{n}, \Sigma^{k} X_{+}^{n}\right]$, then the set of elements corresponding to $M^{n}$ is an orbit under the action of the group $\left[X^{n}, S O(k)\right]=$ $K O^{-1}\left(X^{n}\right)$ on $\left[\Sigma^{k} X_{+}^{n}, \Sigma^{k} X_{+}^{n}\right]$, where $S O(k)$ operates on $S^{k}$ in the right hand $\Sigma^{k} X_{+}^{n}=S^{k} \wedge\left(X_{+}^{n}\right)$.

If $a: S^{n} \rightarrow S O(k)$, and $j: X^{n} \rightarrow S^{n}$ has degree 1 then the result of acting by aj on a map $f: \Sigma^{k} X_{+}^{n} \rightarrow \Sigma^{k} X_{+}^{n}$ is given by

(5.5) Lemma. If a represents $x \in \pi^{n}(S O(k)),[f] \in\left[\Sigma^{k} X_{+}^{n}, \Sigma^{k} X_{+}^{n}\right]$, then $x \cdot[f]=$ $[f]+j^{*} i_{*}(J(x))$ where $i_{*}: \pi_{n+k}\left(S^{k}\right) \rightarrow\left[S^{n+k}, \Sigma^{k} X_{+}^{n}\right]$ is induced by inclusion $S^{k} \subset \Sigma^{k} X_{+}^{n}, j^{*}$ is induced by $\Sigma^{k} j: \Sigma^{k} X^{n} \rightarrow \Sigma^{k} S_{+}^{n}$, and $J: \pi_{n}(S O(k)) \rightarrow \pi_{n+k}\left(S^{k}\right)$ is the J-bomomorphism.

The proof is routine and we omit it.

It follows that if $M_{1}^{n}, M_{2}^{n}$ are smooth homotopy projective spaces, with $N_{1}^{n}$, $N_{2}^{n}$ their (oriented) twofold covers, and $b_{1}, b_{2} \in\left[\Sigma^{k} X_{+}^{n}, \Sigma^{k} X_{+}^{n}\right]$ their spectral cobordism invariants using $X^{n}$ a homotopy projective space, then $M_{1}$ is orientably diffeomorphic to $M_{2}$ implies that $b_{1}$ is in the orbit of $b_{2}$ by $K O^{-1}\left(X^{n}\right)$. If $\tau:\left[\Sigma^{k} X_{+}^{n}, \Sigma^{k} X_{+}^{n}\right] \rightarrow\left[\Sigma^{k} X_{+}^{n}, \Sigma^{k} X_{+}^{n}\right]$ is the involution induced by the generator of order 2 in $K O^{-1}\left(X^{n}\right)$, then $b_{1}=b_{2}$ or $\tau h_{2}$ modulo $j^{*} i_{*}\left(J \pi_{n}(S O(k))\right)$. Thus we have shown

(5.6) Theorem. The spectral cobordism inuariant defines a map from diffeomorphism classes of (oriented) smooth homotopy projective n-spaces to orbits of $\left[\Sigma^{k} X_{+}^{n}, \Sigma^{k} X_{+}^{n}\right]$ by $K O^{-1}\left(X^{n}\right)$, so that different elements $b_{1}, b_{2}$ of $\left[\Sigma^{k} X_{+}^{n}, \Sigma^{k} X_{+}^{n}\right]$ may represent orientably diffeomorphic bomotopy projective spaces if and only if $b_{1}=b_{2}$ or $\tau b_{2}$ modulo $j^{*} i_{*}\left(J \pi_{n}(S O(k))\right.$. Further, if $n \neq-1(4)$ then this condition reduces to $b_{1}=b_{2}$ or $\tau b_{2}$.

(5.7) Lemma. $\left[\Sigma^{k} X_{+}^{n}, \Sigma^{k} X_{+}^{n}\right]=\left[\Sigma^{k} X^{n}, \Sigma^{k} X_{+}^{n}\right]+\left[S^{k}, \Sigma^{k} X_{+}^{n}\right]$.

This is a consequence of the general fact that the map $i_{1}^{*}+i_{2}^{*}:[X \vee Y, Z] \rightarrow$ $[X, Z]+[Y, Z]$ is an isomorphism, where $i_{1}, i_{2}$ are the injections of $X$ and $Y$ in $X \vee Y$.

Now $\left[S^{k}, \Sigma^{k} X_{+}^{n}\right] \cong Z$ and the isomorphism is given by the degree of the homology map. For the spectral cobordism invariants of homotopy equivalences this degree is always 1 , so we may concentrate our attention on the component in $\left[\Sigma^{k} X^{n}, \Sigma^{k} X_{+}^{n}\right]$.

(5.8) Iemma. For k very large $(k>n+1),\left[\Sigma^{k} X^{n}, \Sigma^{k} X_{+}^{n}\right] \cong\left[\Sigma^{k} X^{n}, \Sigma^{k} X^{n}\right]$ $+\left[\Sigma^{k} X^{n}, s^{k}\right]$. 
This follows from the general fact that the map $\pi_{1 *}+\pi_{2 *}:[K, A \times B] \rightarrow$ $[K, A]+[K, B]$ is an isomorphism. For the inclusion $\Sigma^{k} X_{+}^{n}=\Sigma^{k} X^{n} \vee S^{k} C$ $\Sigma^{k} X^{n} \times S^{k}$ induces an isomorphism for the functor $[K$,$] when \operatorname{dim} K<2 k+1$ and, if $k>n+1$, then $\operatorname{dim} \Sigma^{k} X^{n}=k+n<2 k+1$.

In general we may study the group $\left[\Sigma^{k} X_{+}^{n}, \Sigma^{k} X_{+}^{n}\right]$ by applying functors to it, such as homology, or mapping spaces into the left variable $\Sigma^{k} X_{+}^{n}$, or mapping the right variable $\Sigma^{k} X_{+}^{n}$ into another space. For example, take the map $l: X_{+}^{n} \rightarrow P^{\infty}$ which induces the canonical line bundle on $X$, and suspend it, giving a map $\left[\Sigma^{k} X_{+}^{n}, \Sigma^{k} X_{+}^{n}\right] \rightarrow\left[\Sigma^{k} X_{+}^{n}, \Sigma^{k} P^{\infty}\right]$. As $\Sigma^{k} P^{\infty}$ is $k$-connected, $j^{*}:\left[\Sigma^{k} X_{+}^{n}, \Sigma^{k} P^{\infty}\right] \rightarrow$ $\left[\Sigma^{k} X^{n}, \Sigma^{k} P^{\infty}\right]$ is an isomorphism, where $j^{*}$ is induced by the suspension of the inclusion $j: X^{n} \rightarrow X_{+}^{n}$. We denote by $\gamma$ the homomorphism $\gamma:\left[\Sigma^{k} X_{+}^{n}, \Sigma^{k} X_{+}^{n}\right] \rightarrow$ $\left[\Sigma^{k} X^{n}, \Sigma^{k} P^{\infty}\right]$.

(5.9) Lemma. Let $\tilde{p}: N^{n} \rightarrow M^{n}$ be the twofold covering of the smooth bomotopy projective space and let $b(N) \in\left[\Sigma^{k} X_{+}^{n}, \Sigma^{k} X_{+}^{n}\right]$ be the spectral cobordism in. variant of $N$ (using the induced embedding coming from an embedding of $M \times R^{k} C$ $\left.X^{n} \times R^{k}\right)$. Then $\tau b(N)=b(N)$ and $\gamma(b(N))=0$ in $\left[\Sigma^{k} X^{n}, \Sigma^{k} P^{\infty}\right]$.

Proof. We give the proof for $X=P^{n}$, the proof in general being similar. $\tau$ is induced by iaf in the diagram:

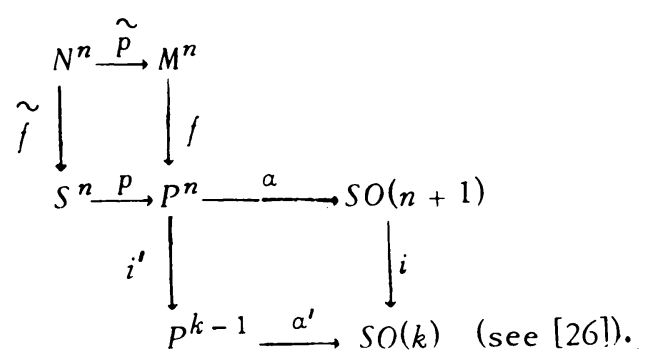

Then the new embedding of $N^{n} \times R^{k} C P^{n} \times R^{k}$ is induced by $i \alpha \tilde{f}=\alpha^{\prime} i^{\prime} p \tilde{f}$.

Since $k \gg n, \pi_{n}\left(P^{k-1}\right)=0$ and thus $i^{\prime} p$ is homotopic to a constant, hence $i a f \tilde{p}$ is homotopic to a constant and the new embedding is isotopic to the original.

Thus $\tau b(N)=b(N)$ 。

Now $b(N)$ is the composite map

$$
\Sigma^{k} P_{+}^{n} \stackrel{c}{\rightarrow} \Sigma^{k} M_{+}^{n} \stackrel{s}{\rightarrow} \Sigma^{k} N_{+} \stackrel{\Sigma^{k} \tilde{p}}{\longrightarrow} \Sigma^{k} M_{+} \stackrel{\Sigma^{k} f}{\longrightarrow} \Sigma^{k} P_{+}^{n}
$$

where $c$ is the spectral orientation map for $M, s$ the Thom-Pontrjagin construction on $N \times R^{k} \subset M \times R^{k}$. Then $\gamma(b(N))$ is the composition with the map $\Sigma^{k} l$ : $\Sigma^{k} P_{+}^{n} \rightarrow \Sigma^{k} P^{\infty}$, where $l: P_{+}^{n} \rightarrow P_{\infty}$. Then $\gamma(b(N))$ is represented by 
$\left(\Sigma^{k} l\right)\left(\Sigma^{k} f\right)\left(\Sigma^{k} \tilde{p}\right) s c$. But since $N$ is 1 -connected, $l \tilde{p}: N_{+} \rightarrow P^{\infty}$ is homotopic to a constant.

Hence $\Sigma^{k}(l \tilde{p})=\left(\Sigma^{k} l\right)\left(\Sigma^{k} f\right)\left(\Sigma^{k} \tilde{p}\right)$ is homotopic to a constant and thus $\gamma b(N)$ $=0$.

(5.11) Lemma. Let $1=$ identity $\epsilon\left[\Sigma^{k} X_{+}^{n}, \Sigma^{k} X_{+}^{n}\right]$. Then $\gamma(1)=\Sigma^{k} l_{n} \epsilon$ $\left[\Sigma^{k} X^{n}, \Sigma^{k} P^{\infty}\right]$.

This is obvious.

Clearly $\tau$ induces an involution (called again $\tau$ ) on $\left[\Sigma^{k} X_{+}^{n}, \Sigma^{k} P^{\infty}\right] \cong$ $\left[\Sigma^{k} X^{n}, \Sigma^{k} P^{\infty}\right]$.

Consider the diagram

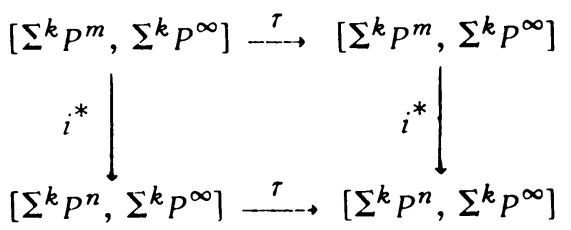

where $i^{*}$ is induced by the suspension of the inclusion $P^{n} \subset P^{m}, m>n$. It follows from the commutativity of (5.10) that (5.12) commutes. Also $i^{*}\left(\Sigma^{k} l_{m}\right)=$ $\Sigma^{k} l_{n}$ where $l_{m}: P^{m} \rightarrow P^{\infty}$ is the inclusion.

Now we recall [28] the following:

(5.13) Theorem (Toda). If $k$ is large, $n$ even, the order of the identity $1 \epsilon$ $\left[\Sigma^{k} P^{n}, \Sigma^{k} \dot{P}^{n}\right]$ is equal to the order of the group $\tilde{K} O\left(P^{n}\right)$.

We recall also from [20]:

(5.14) Theorem. $\tilde{K} O\left(P^{n}\right)$ is a cyclic group of order $2^{s(n)}$, generated by $1-x$, where 1 is the trivial line bundle, $x$ is the canonical line bundle over $P^{n}$. Further $s(n)=$ number of integers $\equiv 0,1,2$, or $4 \bmod 8$ whicb are $\leq n$. (In particular $s(4 k+1)=2 k+1.0)$

(5.15) Corollary. $\left[\Sigma^{k} P^{n}, Y\right]$ is a $Z_{2} s(n)^{-m o d u l e ~ f o r ~ a n y ~ s p a c e ~} Y$, for $n$ even.

Proof. $\left[\Sigma^{k} P^{n}, Y\right]$ is a module over the ring $\left[\Sigma^{k} P^{n}, \Sigma^{k} P^{n}\right]$. Since by (5.13)

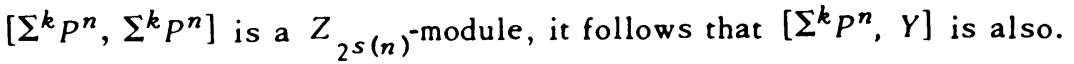

(5.16) Corollary. The order of $l_{n} \in\left[\Sigma^{k} X^{n}, \Sigma^{k} P^{\infty}\right]$ is exactly $2^{s(n)}$.

Proof. By (5.15), $2^{s(n)} l_{n}=0$. On the other hand, $\left(\Sigma^{k} l_{n}\right)^{*}: \tilde{K} O^{-k}\left(\Sigma^{k} P^{\infty}\right) \rightarrow$ $\tilde{K O}^{-k}\left(\Sigma^{k} X^{n}\right), l_{n}^{*}(1)=1, l_{n}^{*}(x)=x(1, x$ being the trivial or canonical line bundles over $X^{n}$ and $\left.P^{\infty}\right)$, and $\left(\Sigma^{k} l_{n}\right)^{*}=\Sigma^{k}\left(l_{n}^{*}\right)$. Hence $\Sigma^{k} l_{n}^{*}$ sends $(x-1) \epsilon$ $K O^{-1}\left(\Sigma^{k} P^{\infty}\right) \cong \tilde{K} O\left(P^{\infty}\right)$ into a generator of $K O^{-k}\left(\Sigma^{k} X^{n}\right) \cong \tilde{K} O\left(P^{n}\right)$, by (5.14). 
Now it is well known that $\left(N\left(\Sigma^{k} l_{n}\right)\right)^{*}=N\left(\Sigma^{k} l_{n}\right)^{*}$, so that $\left(N\left(\Sigma^{k} l_{n}\right)\right)^{*}(x-1)=$ $N(x-1)$. Hence $\left(N\left(\Sigma^{k} l_{n}\right)\right)^{*}(x-1) \neq 0$ for $N<2^{s(n)}$ by $(5.14)$, so that $N\left(\Sigma^{k} l_{n}\right)$ is not nullhomotopic for $N<2^{s(n)}$. Hence order $\Sigma^{k} l_{n}=2^{s(n)}$.

(5.17) Lemma. $\left(\tau \Sigma^{k} l_{n}\right)^{*}= \pm \Sigma^{k} l_{n}^{*}: K O^{-k}\left(\Sigma^{k} P^{\infty}\right) \rightarrow K O^{-1}\left(\Sigma^{k} X^{n}\right)$

Proof. From (5.12) it follows that $\left(i_{*} \tau \Sigma^{k} l_{m}\right)=\tau i_{*} \Sigma^{k} l_{m}=\tau \Sigma^{k} l_{n}$ so that $\left(\tau \Sigma^{k} l_{n}\right)^{*}=\left(i_{*} \tau \Sigma^{k} l_{m}\right)^{*}$ for $m>n$. Since $\tau^{2}=1, K O^{-k}\left(\Sigma^{k} P^{n}\right)=Z_{2 s(n)}^{n}$ and $K O^{-k}\left(\Sigma^{k} P^{m}\right) \rightarrow K O^{-k}\left(\Sigma^{k} P^{n}\right)$ is reduction $\bmod 2^{s(n)}$, and $K O^{-k}\left(\Sigma^{k} P^{m}\right)=$ $\widetilde{K} O\left(P^{m}\right)$ is generated by $l_{m}^{*}(1-x)\left(x\right.$ the canonical line bundle over $\left.P^{\infty}\right)$ it follows that the only involutions of $K O^{-k}\left(\Sigma^{k} P^{n}\right)$ for all $n$ compatible with this situation are \pm 1 , so that the lemma follows.

Using the construction in $\$ 4$, for $M^{m}$ a homotopy projective space, $N$ its (oriented) double covering, consider the normal map

$$
f_{2 r+1}:(2 r+1) M^{m} \cup\left(-r N^{m}\right) \rightarrow M^{m}
$$

given by the identity on each $M^{m}$, the twofold cover on each $-N^{m}$. If $m=4 l+1$ it follows from a theorem of Wall [29] that $f_{2 r+1}$ is normally cobordant to a homotopy equivalence $g_{2 r+1}: M^{m}(2 r+1) \rightarrow M^{m}$.

(5.18) Theorem. For $0 \leq r<s \leq 2^{2 l-1}, M^{4 l+1}(2 r+1)$ is not normally cobordant to $M^{4 l+1}(2 s+1)$ (and a fortiori not orientably diffeomorphic).

Proof. By (4.5), the spectral cobordism invariant $b(M(2 r+1))=1+$ $r(2(1)-a) \in\left[\Sigma^{k} M_{+}^{m}, \Sigma^{k} M_{+}^{m}\right]$, where $1=$ identity, $\alpha=$ spectral cobordism invariant of the twofold cover $N^{m} \rightarrow M^{m}$. By (5.9), $\gamma(\alpha)=0$, so $\gamma b(M(2 r+1))=$ $(2 r+1) \Sigma^{k} l_{m} \in\left[\Sigma^{k} M^{m}, \Sigma^{k} P^{\infty}\right]$. If $M(2 r+1)$ and $M(2 s+1)$ are orientably diffeomorphic by $(5.6)$, either $b(M(2 r+1))=b(M(2 s+1))$ or $\tau h(M(2 s+1))$. By (5.17), $\tau \Sigma^{l} l_{m}^{*}= \pm \Sigma^{k} l_{m}^{*}$, so that

$$
(\gamma b(M(2 r+1)))^{*}=(2 r+1) \Sigma^{k} l_{m}^{*}= \pm(\gamma b(M(2 s+1)))^{*}= \pm(2 s+1) \Sigma^{k} l_{m}^{*} .
$$

Since $l_{m}^{*}(1-x)$ generates $K O\left(P^{m}\right)=K O^{-k}\left(\sum^{k} M^{m}\right) \cong Z_{22 l+1}$, it follows that this is impossible for $0 \leq r<s \leq 2^{2 l-1}$.

Note that by (5.16), $\Sigma^{\bar{k}} l_{m}^{*}$ has order $2^{2 k+1}$ in $\left[\Sigma^{k} M^{m}, \Sigma^{k} P^{\infty}\right]$, so that one cannot improve (5.18) without studying more delicate invariants, e.g. the part of the spectral cobordism invariant in $\left[\Sigma^{k} M, S^{k}\right]$.

One may use the same construction in other dimensions, but there will be a surgery obstruction which is the Kervaire invariant (see (4.1)) if $m=2 q$, or the Kervaire invariant of a codimension 1 submanifold if $m=4 l+3$ (see proof of (7.1)). These obstructions are zero if and only if $2 r+1 \equiv \pm 1$ (8) (see (4.8)) so we get, by a similar argument as above, 
(5.19) Theorem. For $m \equiv 1(4),(2 r+1) M^{m} \cup\left(-r N^{m}\right) \rightarrow M^{m}$ is normally cobordant to a bomotopy equivalence $g_{2 r+1}: M^{m}(2 r+1) \rightarrow M^{m}$ if and only if $2 r+1 \equiv \pm 1$ (mod 8$)$, and different values of $r$ give at least $2^{s(m)-3}$ orientably nondiffeomorphic bomotopy projective spaces.

6. Involutions on certain Brieskorn spheres, smooth and PL classifications. Let us consider the equation

$$
z_{0}^{d}+z_{1}^{2}+z_{2}^{2}+\cdots+z_{2 q+1}^{2}=0
$$

for $z_{i} \in \mathrm{C}$, the complex numbers and $d$ odd. Then (6.1) defines an affine algebraic variety with the origin as the unique singularity, of real dimension $4 q+2$. The intersection of the locus of (6.1) with the unit ball:

$$
\|z\|=\sum z_{i} \bar{z}_{i}=1
$$

gives a smooth manifold $\Sigma_{d}^{4 q+1}$ of dimension $4 q+1$. This manifold is a homotopy sphere. The possibility of representing homotopy spheres in this way was discovered by Brieskorn, and these representations are called Brieskorn spheres. They have been studied by him and others including Hirzebruch and Milnor. A beautiful account of this theory is given in [20].

The algebraic equations defining $\Sigma_{d}^{4 q+1}$ have natural symmetry which gives naturally many groups acting smoothly on $\Sigma_{d}^{4 q+1}$, which have been studied by many authors (e.g. [17], [6]). Here we shall study the natural fixed point free involution $T: \Sigma_{d}^{4 q+1} \rightarrow \Sigma_{d}^{4 q+1}$ given by

$$
T\left(z_{0}, \cdots, z_{2 q+1}\right)=\left(z_{0},-z_{1},-z_{2}, \ldots,-z_{2 q+1}\right) .
$$

Clearly, if $z=\left(z_{0}, \cdots, z_{2 q+1}\right)$ is fixed under $T$, then $z_{1}=z_{2}=\cdots=z_{2 q+1}=0$ so that, from (6.1), $z_{0}=0$ also, and $z=0$. Hence $T$ is without fixed points on $\Sigma_{d}^{4 q+1}$. These involutions were studied in the paper of Atiyah-Bott [4], and we shall recapture and extend their results on smooth classification of them using our methods. We shall also calculate the Kervaire invariants of them. Related work has been done by Giffen ([13], [14]).

(6.4) Theorem. $\Sigma_{d}^{4 k+1} / T$ is normally cobordant to $P^{4 k+1}(d)$. (See $\$ 4$ for the definition of $P^{4 k+1}(d)$.)

Proof. If we modify (6.1) to

$$
P(z)=z_{0}^{d}+z_{1}^{2}+\cdots+z_{2 k+1}^{2}=\epsilon, \quad \epsilon \text { small, real, }
$$

and consider

$$
\|z\|=\sum_{z_{i} \bar{z}_{i} \leq 1}
$$


we get a manifold $W^{4 k+2} \subset \mathbf{C}^{2 k+2}$, and it is easy to see that $\partial W$ is equivariantly diffeomorphic to $\Sigma_{d}$, with $T$ defined by (6.3).

Now (6.3) defines an involution $\bar{T}: W \rightarrow W$ which has fixed points $F=$ $\left\{(\lambda, 0, \ldots, 0) \in W\right.$, where $\left.\lambda^{d}=\epsilon\right\}$.

Consider $N=\mathbf{C} \times D^{4 k+2} \subset \mathbf{C}^{2 k+2}, N=\left\{\left(z_{0}, \cdots, z_{2 k+1}\right)\right.$ such that $\left.\Sigma_{i>0} z_{i} \bar{z}_{i}<\delta\right\}$ where $\delta \ll \epsilon, \delta$ real. Then $N$ is invariant under $T$ and $N^{\prime}=$ $N \cap W=d$ disjoint disks, a neighborhood of $F$. Then $\bar{T} \mid W-N^{\prime}$ is fixed point free.

Since $\bar{T}$ is smooth, $\bar{T} \mid N^{\prime}$ is smoothly equivalent to a linear involution, and since it is free on $\partial N^{\prime}$ it follows that $\bar{T} \mid N^{\prime}$ is smoothly equivalent to the antipodal map on each disk. Hence $\left(W-N^{\prime}\right) / T$ is a cobordism of $\Sigma_{d} / T$ with $d P^{4 k+1}=\partial N^{\prime} / \bar{T}$.

Now $W-N^{\prime} \subset \mathbf{C}^{2 k+1}-\mathbf{C} \times \overline{0}$, where $W-N^{\prime}=P^{-1}(t) \cap\left(D^{4 k+4}-N\right), \overline{0}=$ $(0, \ldots, 0) \in \mathbf{C}^{2 k}, P: \mathbf{C}^{2 k+1} \rightarrow \mathbf{C}, t$ is a regular value. Since $P$ is invariant under $\bar{T}, P \bar{T}=P$, it follows that $P$ induces $P^{\prime}: \mathbf{C}^{2 k+1}-\mathbf{C} \times \overline{0} / \bar{T} \rightarrow \mathbf{C}$ and $\left(W-N^{\prime}\right) / \bar{T}$ $=P^{\prime-1}(t) \cap V$, where $V=D^{4 k+4}-N$. Since $\bar{T}\left(z_{0}, z_{1}, \cdots, z_{2 k+1}\right)=\left(z_{0},-z_{1}, \ldots\right.$, $\left.-z_{2 k+1}\right), \mathbf{C}^{2 k+1}-\mathrm{C} \times \overline{0} / \bar{T}=P^{4 k+1} \times R^{3}$. Since $\left(W-N^{\prime}\right) / \bar{T}=P^{\prime-1}(t) \cap V$ and $t$ is a regular value of $P^{\prime},\left(W-N^{\prime}\right) / \bar{T}$ has a trivial normal bundle in $P^{4 k+1} \times R^{3}$, and hence it defines a "normal" cobordism between $\Sigma_{d} / T$ and $d P^{4 k+1}$, except that the inclusion maps of the boundary components into $P^{4 k+1}$ have degree $d$. Take the 2-fold cover $p: S^{4 k+1} \rightarrow P^{4 k+1}$ and make it regularly homotopic to an embedding $a_{:} S^{4 k+1} \times I \times R^{t-1} \rightarrow P^{4 k+1} \times R^{t}, t$ very large. This has degree 2 so if we take a connected sum along the boundary $\Sigma_{d} / T$ of $\left(W-N^{\prime}\right) / T$ with $(-l) S^{4 k+1} \times I \times R^{t-1}$ we get a normal cobordism of $\Sigma_{d} / T \#\left(-l S^{4 k+1}\right)=\Sigma_{d} / T$ with $P^{4 k+1}(d)$, which completes the proof of (6.4).

Applying (5.18) we obtain a different proof of the result of Atiyah-Bott [4]:

(6.5) Theorem. On the Brieskorn spheres $\Sigma_{d}^{4 k+1}$, with involution $T: \Sigma_{d} \rightarrow$ $\Sigma_{d}, \Sigma_{d} / T_{d}$ is not normally cobordant to and bence not diffeomorpbic to $\Sigma_{d^{\prime}} / T_{d^{\prime}}$ if $0 \leq r<s \leq 2^{2 k-1}, d=2 r+1, d^{\prime}=2 s+1$.

Giffen [14] has improved this result to $2^{2 k}$, for which we also have a proof, which will be given elsewhere.

Applying (4.15), (4.16), (4.17), noting that $\Sigma_{d} / T$ is normally cobordant to $P^{4 k+1}(d)$, we get

(6.6) Theorem.

$$
\begin{aligned}
k_{2 i}\left(\Sigma_{d}^{4 k+1} / T\right) & =1 \quad \text { if } d \equiv \pm 3(8) \\
& =0
\end{aligned}
$$


and thus $T$ admits bomotopy l-spheres as characteristic submanifolds if and only if $d \equiv \pm 1$ (8) or $l \equiv 1$ (4).

(This has also been proved by Giffen [13].)

From (4.18) we deduce a result of [20]:

(6.7) Theorem. The Brieskorn sphere $\Sigma_{d}^{4 k+1}$ bounds a manifold of Kervaire invariant 1 if $d \equiv \pm 3(8)$ and of Kervaire invariant 0 if $d \equiv \pm 1$ (8). Hence $\Sigma_{d}^{4 k+1}$ generates $b P^{4 k+2}$ for $d \equiv \pm 3$ (8) and $\Sigma_{d}^{4 k+1}=S^{4 k+1}$ for $d \equiv \pm 1$ (8).

7. Embedding homotopy projective spaces. As an application of $\$ \S 2,3$, and 4 , and the results of [9] we give a theorem for finding embeddings of homotopy real projective spaces. This theorem grew out of conversations with E. Rees and is applied by him in [24] in the case where $n=7$ and $\xi^{k}=\epsilon^{2}$.

(7.1) Theorem. Suppose $\xi^{k}$ is a $k$-plane bundle over $P^{n}, n \geq 5, k \geq 2$. Suppose $p^{*}(\xi)$ is fibre bomotopy trivial, where $p: S^{n} \rightarrow P^{n}$ is the 2-fold cover and let $a \in \pi_{n+k}(T(\xi))$ be such that $b(\alpha) \cap U=\left[P^{n}\right] \in H_{n}\left(P^{n} ; Z_{2}\right)$, where $b$ is the Hurewicz bomomorphism, $U \in H^{k}\left(T(\xi) ; Z_{2}\right)$ the Thom class, and $\left[P^{n}\right]$ the funda. mental class. Then there is a smooth $M^{n} \subset S^{n+k+1}$ and a bomotopy equivalence $f: M^{n} \rightarrow P^{n}$ with the normal bundle of $M$ equal to $f^{*}(\xi)+\epsilon^{1}$.

Proof. Since $p^{*} \xi$ is fibre homotopy trivial, there is a $\beta \in \pi_{n+k}\left(T\left(p^{*}(\xi)\right)\right)$ such that $b(\beta) \cap U_{p^{*} \xi}=\left[S^{n}\right]$. Making $\beta$ t-regular, we get a manifold $Q^{n} \subset S^{n+k}$ with normal bundle $\nu_{Q}$ and a normal map $s: Q^{n} \rightarrow S^{n}$, e: $\nu_{Q} \rightarrow p^{*} \xi$. By using surgery, we may assume $Q^{n}$ is 1 -connected. If the Kervaire invariant of this normal map (i.e. $k(Q)$ with the orientation induced by $e$ ) is nonzero, we can get another normal map $s \# s: Q \# Q \rightarrow S^{n} \# S^{n}=S^{n}$ etc., and $k(Q \# Q)=0$. The composite with $p: S^{n} \rightarrow P^{n}$, and a map $p^{*} \xi \rightarrow \xi$ gives us a map of degree $2, p^{\prime}: Q^{\prime} \rightarrow P^{n}$, covered by $e^{\prime}: \nu_{Q^{\prime}} \rightarrow \xi$, where $Q^{\prime} \subset S^{n+k}$ with normal bundle $\nu_{Q^{\prime}}$, and $k\left(Q^{\prime}\right)=0$. Then $\left(p^{\prime}, e^{\prime}\right)$ represents a class $\beta^{\prime} \in \pi_{n+k}(T(\xi))$, and since $Q^{\prime}$ is 1 -connected, $p^{\prime *}=0$ on $H^{1}\left(P^{n} ; Z_{2}\right)$ and hence $p^{\prime *} \equiv 0$.

Now by making $\alpha_{:} S^{n+k} \rightarrow T(\xi)$ t-regular on $P^{n}$ we get a map $g: N^{n} \rightarrow P^{n}$ covered by a bundle map $b: \nu_{N} \rightarrow \xi$, where $\nu_{N}$ is the normal bundle of $N$ in $S^{n+k}$. Now degree $g \equiv 1$ (2) from the condition $b(\alpha) \cap U=\left[P^{n}\right]$ in $H_{n}\left(P^{n} ; Z_{2}\right)$, so by taking the disjoint union with a number of copies of the normal map $p^{\prime}$ : $Q^{\prime} \rightarrow P^{n}$ we obtain a normal map (i.e. of degree 1) $g^{\prime}: N^{\prime} \rightarrow P^{n}, b^{\prime}: \nu_{N^{\prime}} \rightarrow \xi$, represented by an element $\alpha^{\prime} \in \pi_{n+k}(T(\xi))$, where $\alpha^{\prime}=\alpha+\beta^{\prime}$.

By the corollary of [9], if $\alpha^{\prime} \in \pi_{n+k}(T(\xi))$ suspends to the "normal invariant" of some homotopy projective space $M$, then $M \subset S^{n+k+1}$ with normal bundle in. duced from $\xi+\epsilon^{1}$ by the homotopy equivalence. But this is equivalent to the statement that the normal map into $P^{n}$ represented by $\alpha^{\prime}$ is normally cobordant to a homotopy equivalence, i.e., that its surgery obstruction is zero, if $n \geq 5$. 
From the results of Wall [29] there is no surgery obstruction if $n \equiv 1$ (4) so in that case we are finished. If $n=2 q$, the surgery obstruction is the Kervaire invariant $k\left(N^{\prime}\right)$ (see (4.1)). Hence if $k\left(N^{\prime}\right) \neq 0$, take the new normal map $\bar{g}: 3 N^{\prime} \cup\left(-Q^{\prime}\right) \rightarrow$ $P^{n}$, etc. $\bar{g}=3 g^{\prime} \cup p^{\prime}$.

Since $p^{\prime *} \equiv 0$, it follows from (2.13) that $k\left(3 N^{\prime} \cup\left(-Q^{\prime}\right)\right)=k\left(3 N^{\prime}\right)+k\left(Q^{\prime}\right)=$ $k\left(3 N^{\prime}\right)$ since $Q^{\prime}$ was constructed to have $k\left(Q^{\prime}\right)=0$. Then by $(4.8), k\left(3 N^{\prime}\right)=$ $k\left(N^{\prime}\right)+1=0$, so that the surgery obstruction is zero for the normal map $\bar{g}$ and the proof is finished as before using [9].

If $n \equiv-1(4)$, then we use the same process as above to construct $\bar{\alpha} \epsilon$ $\pi_{n+k}(T(\xi))$ so that the normal map induced into $P^{n-1}$ has surgery obstruction zero. The remainder of the map is a normal map into a disk $D^{n}$ which is a homotopy equivalence on the boundary, so it can be made normally cobordant to a homotopy equivalence leaving the boundary fixed. Thus this normal map into $P^{n}$ is normally cobordant to a homotopy equivalence (compare the proof of [9] and of (5.19)), and again we are done.

\section{BIBLIOGRAPHY}

1. J. F. Adams, On the groups $J(X)$. II, Topology 3 (1965), 137-171. MR 33 \#6626.

2. - Vector fields on spheres, Ann. of Math. (2) 75 (1962), 603-632.

MR $25 \# 2614$.

3. M. F. Atiyah, Thom complexes, Proc. London Math. Soc. (3) 11 (1961), 291-310. MR 24 \#A 1727.

4. M. F. Atiyah and R. Bott, A Lefschetz fixed point formula for elliptic complexes. II. Applications, Ann. of Math. (2) 88 (1968), 451-491. MR $38 \# 731$.

5. I. Berstein, Involutions with nonzero Arf invariant, Bull. Amer. Math. Soc. 74 (1968), 678-682. MR 38 \#5225.

6. G. Bredon, Exotic actions on spheres, Proc. Conf. on Transformation Groups (New Orleans, La., 1967), Springer, New York, 1967, pp. 47-76. MR 42 \#1146.

7. W. Browder, The Kervaire invariant of framed manifolds and its generalization, Ann. of Math. (2) 90 (1969), 157-186. MR 40 \#4963.

8. - Surgery on simply connected manifolds, Springer-Verlag, Berlin and New York, 1972.

9. ——, Embedding smooth manifolds, Proc. Internat. Congress Math. (Moscow, 1966), "Mir", Moscow, 1968. MR 38 \#6611.

10. W. Browder and G. R. Livesay, Fixed point free involutions on homotopy spheres, Bull. Amer. Math. Soc. 73 (1967), 242-245. MR 34 \#6781.

11. E. H. Brown, Jr., The Arf invariant of a manifold, Conf, on Algebraic Topology (Univ. of Illinois at Chicago Circle, Chicago, Ill., 1968), Univ. of Illinois at Chicago Circle, Chicago, Ill., 1969, pp. 9-18. MR $40 \# 4964$.

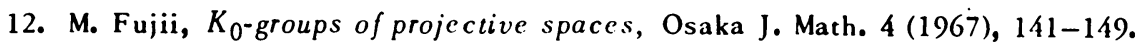
MR $36 \# 2143$.

13. C. H. Giffen, Desuspendability of free involutions on Brieskorn spheres, Bull. Amer. Math. Soc. 75 (1969), 426-429. MR 39 \#2168. 
14. C. H. Giffen, Smooth homotopy projective spaces, Bull. Amer. Math. Soc. 75 (1969), 509-513. MR 39 \#964.

15. A. Haefliger and V. Poenaru, La classification des immersions combinatoires, Inst. Haute Études Sci. Publ. Math. No. 23 (1964), 75-91. MR 30 \#2515.

16. M. W. Hirsch, On the fibre homotopy type of normal bundles, Michigan Math. J. 12 (1965), 225-229. MR $32 \# 1724$.

17. F. Hirzebruch and K. H. Mayer, $O(n)$-Mannigfaltigkeiten, exotische Sphären und Singularitäten, Lecture Notes in Math., no. 57, Springer-Verlag, Berlin and New York, 1968. MR 37 \#4825.

18. S. López de Medrano, Some results on involutions of homotopy spheres, Proc. Conf. on Transformation Groups (New Orleans, La., 1967), Springer, New York, 1968, pp. 167-174. MR 40 \#6564.

19. J. Milnor, Microbundles and differentiable structures, Princeton University, Princeton, N. J., 1961 (mimeographed notes).

20. - Singular points of complex hypersurfaces, Ann. of Math. Studies, no. 61, Princeton Univ. Press, Princeton, N. J.; Univ. of Tokyo Press, Tokyo, 1968. MR 39 \#969.

21. D. Montgomery and C. T. Yang, Free differentiable actions on homotopy spheres, Proc. Conf. on Transformation Groups (New Orleans, La., 1967), Springer, New York, 1968, pp. 175-192. MR 39 \#6354.

22. C. Morlet, Les voisinages tubulaires des variétés semi-linéaires, C. R. Acad. Sci. Paris Sér. A-B 262 (1966), A740-A743. MR 35 \#4930a.

23. P. Olum, Mappings of manifolds and the notion of degree, Ann. of Math. (2) 58 (1953), 458-480. MR 15, 338.

24. E. Rees, Embeddings of real projective spaces, (to appear).

25. C. P. Rourke and B. J. Sanderson, Block bundles. I, II, III, Ann. of Math. (2) 87 (1968), 1-28, 256-278, 431-483. MR 37 \#2234a, b; MR 38 \#29.

26. N. E. Steenrod and D. B. A. Epstein, Cohomology operations, Ann. of Math. Studies, no. 50, Princeton Univ. Press, Princeton, N. J., 1962: MR 26 \#3056.

27. D. Sullivan, Triangulating homotopy equivalences, Ph. D. Thesis, Princeton University, Princeton, N. J., 1966.

28. H. Toda, Order of the identity class of a suspension space, Ann. of Math. (2) 78 (1963), 300-325. MR 27 \#6271.

29. C. T. C. Wall, Surgery on compact manifolds, Academic Press, London, 1971.

30. - Free piecewise linear involutions on spheres, Bull. Amer. Math. Soc. 74 (1968), 554-558. MR 36 \#5955.

DEPARTMENT OF MATHEMATICS, PRINCETON UNIVERSITY, PRINCETON, NEW JERSEY 08540 